The Astrophysical Journal, 612:168-190, 2004 September 1

(C) 2004. The American Astronomical Society. All rights reserved. Printed in U.S.A.

\title{
A LARGE STELLAR EVOLUTION DATABASE FOR POPULATION SYNTHESIS STUDIES. I. SCALED SOLAR MODELS AND ISOCHRONES
}

\author{
Adriano Pietrinferni ${ }^{1}$ \\ INAF Osservatorio Astronomico di Teramo, Via M. Maggini, I-64100 Teramo, Italy; pietrinferni@te.astro.it \\ SANTI CASSISI ${ }^{2}$ \\ INAF Osservatorio Astronomico di Teramo, Via M. Maggini, I-64100 Teramo, Italy; cassisi@te.astro.it
}

Maurizio Salaris

Astrophysics Research Institute, Liverpool John Moores University, Twelve Quays House, Birkenhead CH41 1LD, UK; ms@astro.livjm.ac.uk

AND

Fiorella Castelli ${ }^{3}$

Istituto di Astrofisica Spaziale e Fisica Cosmica, CNR, Via del Fosso del Cavaliere, I-00133 Rome, Italy; castelli@ts.astro.it Received 2004 January 29; accepted 2004 May 6

\begin{abstract}
We present a large and updated stellar evolution database for low-, intermediate-, and high-mass stars in a wide metallicity range, suitable for studying Galactic and extragalactic simple and composite stellar populations using population synthesis techniques. The stellar mass range is between $\sim 0.5$ and $10 M_{\odot}$ with a fine mass spacing. The metallicity $[\mathrm{Fe} / \mathrm{H}]$ comprises 10 values ranging from -2.27 to 0.40 , with a scaled solar metal distribution. The initial He mass fraction ranges from $Y=0.245$, for the more metal-poor composition, up to 0.303 for the more metal-rich one, with $\Delta Y / \Delta Z \sim 1.4$. For each adopted chemical composition, the evolutionary models have been computed without (canonical models) and with overshooting from the Schwarzschild boundary of the convective cores during the central H-burning phase. Semiconvection is included in the treatment of core convection during the He-burning phase. The whole set of evolutionary models can be used to compute isochrones in a wide age range, from $\sim 30 \mathrm{Myr}$ to $\sim 15 \mathrm{Gyr}$. Both evolutionary models and isochrones are available in several observational planes, employing an updated set of bolometric corrections and color- $T_{\text {eff }}$ relations computed for this project. The number of points along the models and the resulting isochrones is selected in such a way that interpolation for intermediate metallicities not contained in the grid is straightforward; a simple quadratic interpolation produces results of sufficient accuracy for population synthesis applications. We compare our isochrones with results from a series of widely used stellar evolution databases and perform some empirical tests for the reliability of our models. Since this work is devoted to scaled solar chemical compositions, we focus our attention on the Galactic disk stellar populations, employing multicolor photometry of unevolved field main-sequence stars with precise Hipparcos parallaxes, well-studied open clusters, and one eclipsing binary system with precise measurements of masses, radii, and $[\mathrm{Fe} / \mathrm{H}]$ of both components. We find that the predicted metallicity dependence of the location of the lower, unevolved main sequence in the color magnitude diagram (CMD) appears in satisfactory agreement with empirical data. When comparing our models with CMDs of selected, well-studied, open clusters, once again we were able to properly match the whole observed evolutionary sequences by assuming cluster distance and reddening estimates in satisfactory agreement with empirical evaluations of these quantities. In general, models including overshooting during the H-burning phase provide a better match to the observations, at least for ages below $\sim 4$ Gyr. At $[\mathrm{Fe} / \mathrm{H}]$ around solar and higher ages (i.e., smaller convective cores) before the onset of radiative cores, the selected efficiency of core overshooting may be too high in our model, as well as in various other models in the literature. Since we also provide canonical models, the reader is strongly encouraged to always compare the results from both sets in this critical age range.
\end{abstract}

Subject headings: galaxies: stellar content — Galaxy: disk — open clusters and associations: general stars: evolution - stars: horizontal-branch

\section{INTRODUCTION}

The availability of large sets of stellar evolution models spanning a wide range of stellar masses and initial chemical

\footnotetext{
${ }^{1}$ Dipartimento di Statistica, Università di Teramo, Loc. Coste S. Agostino, 64100 Teramo, Italy.

${ }_{2}$ Instituto de Astrofísica de Canarias, 38200 La Laguna, Tenerife, Canary Islands, Spain.

${ }^{3}$ INAF-Osservatorio Astronomico di Trieste, via Tiepolo 11, 34131 Trieste, Italy; castelli@ts.astro.it.
}

compositions is a necessary prerequisite for any investigation aimed at interpreting observations of Galactic and extragalactic, resolved and unresolved stellar populations. A reliable library of stellar evolution models used to study stellar populations in various environments has to satisfy at least three main criteria: (1) the input physics has to be up to date and the treatment of physical processes has to be adequate and as much accurate as possible, (2) the set of models has to be homogeneous, in the sense that all evolutionary phases and initial chemical compositions have to be computed with the same evolutionary code and the same physical framework, and 
(3) the models have to reproduce as many empirical constraints as possible.

The accuracy of the results obtained from methods based on stellar evolution models is strongly dependent on the reliability of the physical framework adopted in the computations. In this respect, it is very interesting to note that only about a decade ago the available stellar models were already producing models that matched several observational constraints reasonably well. However, new results from helioseismology came to show that solar models available at that time were seriously challenged by these new empirical results. This stimulated a large effort toward improving our knowledge of the physics of stellar matter, and as a consequence, in these last years, several aspects of stellar physics (e.g., radiative opacities, equation of state [EOS]) have been largely improved. This process has produced a continuous improvement in solar models as well as in the whole stellar evolution field, with nonnegligible variations in quantitative predictions, as in the case of the Galactic globular cluster ages (e.g., Chaboyer \& Kim 1995; Mazzitelli et al. 1995; Salaris et al. 1997). A detailed discussion of the effect of recent improvements in the input physics on the stellar evolution results can be found in Cassisi et al. (1998). This evidence provides a clear support for the need of a continuous improvement and update of stellar model databases.

The homogeneity of the adopted model library is also extremely important, because the use of different sets of models (computed by different authors with different input phsyics) for different evolutionary phases and/or chemical compositions and/or mass ranges can introduce subtle inconsistencies in the interpretation of observations and can affect the predictive power of stellar population synthesis techniques.

As for the comparison with empirical constraints, it goes without saying that this is the best way to assess the reliability of the chosen evolutionary scenario. In particular, it is very important to compare theoretical models with empirical constraints from resolved stellar populations, such as Galactic field stars and Galactic stellar clusters. This is a fundamental step in order to be able to estimate the level of accuracy of the models, as well as for evaluating the uncertainties that could affect the population synthesis analysis based on the same models.

The need of continuously improved stellar evolution databases (in spite of the already existing large number of stellar models and isochrone sets) incorporating the latest advances in modeling the stellar physics cannot be more emphasized by the fact that existing differences among stellar model libraries translate into large uncertainties in, for example, the ages of external galaxies; this is well illustrated by, for example, the large differences obtained by Spinrad et al. (1997) and Yi et al. (2000) regarding the age (at least 3.5 Gyr vs. 1.4-1.8 Gyr, respectively) of an unresolved high-redshift galaxy (LBDS 53W091) due mainly to large differences in the colors (and spectra) predicted by the two different sets of isochrones used in the two aforementioned papers. Such large differences due only to uncertainties in the modeling of stellar populations have important consequences for our interpretation of the epoch and mechanisms of galaxy formation.

Only by employing independent sets of stellar model databases can one assess the real uncertainties in the inferred parameters for resolved and unresolved stellar populations. In the case of some key stellar input physics, such as boundary conditions, overshooting, atomic diffusion efficiency, and mass loss, it is not yet clear what the best prescription is; to this one has to add current uncertainties on the synthetic spectra used to transform the output of theoretical stellar models into observed quantities such as colors, magnitudes, and line strengths. It is therefore important that several sets of models be available, computed with the best possible inputs for the well-established physics (e.g., EOS, opacities), and with various choices of the parameters describing the physical processes not yet well understood (e.g., mixing, convection, and mass loss).

The recent literature is rich with updates of stellar model libraries; see, for example, Bono et al. (2000), Girardi et al. (2000), VandenBerg et al. (2000), Lejeune \& Schaerer (2001), Yi et al. (2001), and Castellani et al. (2003). However, not all of them cover homogeneously a large range of ages and chemical compositions. Over the years, we have published a number of specialized papers with a series of specific goals, other than addressing the issue of population synthesis techniques. We have investigated the effects of improvements in the adopted physical inputs on stellar models (Salaris \& Cassisi 1996; Brocato et al. 1998; Cassisi et al. 1998, 1999 and references therein); we have compared our low-mass isochrones with selected observational constraints from old stars (e.g., Cassisi \& Salaris 1997; Salaris \& Cassisi 1998; Riello et al. 2003) and obtained estimates of Galactic globular cluster ages (e.g., Salaris et al. 1997; Salaris \& Weiss 1998, 2002; Weiss \& Solaris 1999), initial He abundance of globular cluster stars (Cassisi et al. 2003; Salaris et al. 2004), and pulsational properties of variable stars (De Santis \& Cassisi 1999; Bono et al. 1997). This notwithstanding, we have never published an extended and homogeneous set of stellar models covering a large range of stellar masses, chemical compositions, and evolutionary phases suitable for population synthesis analysis as well as for any other kind of investigation making extensive use of stellar evolution models.

The main purpose of this work is to fill this gap, by providing an up-to-date and complete set of stellar models for both low-, intermediate-, and high-mass stars up to $10 M_{\odot}$, spanning a large metallicity regime from metal-poor star systems to super-metal-rich populations. Our updated theoretical models are then coupled to a new set of color transformation and bolometric corrections. In this paper, all theoretical results are based on a scaled solar metal distribution. The case of an $\alpha$-element enhanced metal mixture will be the subject of a forthcoming paper.

The paper is organized as follows: $\S 2$ summarizes the improvements made to our evolutionary code, while $\S 3$ deals specifically with the difficult problem of the core convection treatment. Our standard solar model is briefly discussed in $\S 4$, and the model library is presented in $\S 5$. Comparisons with the most used existing isochrone databases, and with selected empirical constraints, are discussed in $\S \S 6$ and 7, respectively. A summary and conclusions follow in $\S 8$.

\section{THE STELLAR EVOLUTION CODE AND INPUT PHYSICS}

The stellar evolution code adopted in this work is the same used by Cassisi \& Salaris (1997) and Salaris \& Cassisi (1998), with various updates. We have added the nuclear burning of light elements such as $\mathrm{Li}, \mathrm{B}$, and $\mathrm{Be}$, improved the numerical procedures that optimize the choice of both the number of mesh points within a model and the evolutionary time steps, and updated the model input physics. In the following we summarize our choice of the most relevant input physics, and - in case of differences with our previous Cassisi \& Salaris (1997) models - we discuss its impact on relevant model parameters. 
1. The radiative opacities are from the OPAL tables (Iglesias \& Rogers 1996) for temperatures larger than $10^{4} \mathrm{~K}$, whereas the opacities by Alexander \& Ferguson (1994), which include the contribution from molecules and grain, have been adopted for lower temperatures. Both high- and low-temperature opacity tables assume a scaled solar heavy-element distribution (Grevesse \& Noels 1993). As for the electron conduction opacities we use the recent results of Potekhin et al. (1999) and Potekhin (1999, hereafter P99). These computations also cover the physical conditions typical of degenerate He cores in red giant branch (RGB) stars; this avoids the extrapolation we had to perform with our previous choice of the Itoh et al. (1983, 1993, hereafter 193) conductive opacities since, as first shown by Catelan et al. (1996), the I93 opacities did not cover the degenerate He cores in low-mass stars. Before computing the whole set of evolutionary models presented in this work, we have tested the effect of the P99 opacity on the size of electron degenerate $\mathrm{He}$ cores at the He flash ignition: at a metallicity $Z=0.002$ the change of the He core mass at the He flash in a $0.8 M_{\odot}$ model is equal to $6 \times 10^{-4} M_{\odot}$, in the sense that the P99 opacities produce a less massive He core with respect to the I93 opacities, but at solar composition the trend reverses, the He core mass being $\approx 7 \times 10^{-4} M_{\odot}$ larger when using the P99 opacity. It is clear that the effect related to the use of the P99 data is not large. This notwithstanding, we decided to update our code by using the P99 opacities, in order to avoid any extrapolation.

2. We updated the energy loss rates for plasma-neutrino processes (relevant in the He degenerate cores) using the most recent and accurate results provided by Haft et al. (1994). For all other processes we still rely on the same prescriptions adopted by Cassisi \& Salaris (1997). In the case of a $1 M_{\odot}$ star with $Z=0.002$, these new neutrino rates produce an He core mass at the He flash $\sim 0.005 M_{\odot}$ larger [and the corresponding $\log \left(L / L_{\odot}\right)$ is increased by $\left.\sim 0.025\right]$ than our previous results based on the Munakata et al. (1985) rates. However, the same $1 M_{\odot}$ star at solar metallicity (see $\S 4$ ) shows an He core mass at the He flash decreased by $0.003 M_{\odot}$ [the corresponding $\log \left(L / L_{\odot}\right)$ is decreased by $\left.\sim 0.015\right]$ with respect to our previous models.

3. The nuclear reaction rates have been updated by using values from the NACRE database (Angulo et al. 1999), with the exception of the ${ }^{12} \mathrm{C}(\alpha, \gamma){ }^{16} \mathrm{O}$ reaction. For this reaction we employ the more accurate recent determination by Kunz et al. (2002). Electron screening is treated according to Graboske et al. (1973). We have tested the effect on the main-sequence (MS) turnoff (TO) luminosity when passing from our previously adopted rates (Caughlan \& Fowler 1988) to the NACRE ones. We found that for a $1 M_{\odot}$ star of solar composition the age at the TO increases by $0.6 \%$, and $\log \left(L / L_{\odot}\right)$ at the TO increases by $\sim 3.5 \%$.

The new ${ }^{12} \mathrm{C}(\alpha, \gamma){ }^{16} \mathrm{O}$ rate by Kunz et al. (2002) reduces the $\mathrm{He}$ burning evolutionary lifetimes (at a fixed He core mass and envelope composition) by approximately $7 \%-8 \%$, with respect to the results obtained with our previously adopted Caughlan et al. (1985) rate.

4. The detailed EOS by A. Irwin ${ }^{4}$ has been used. A full description of this EOS is still in preparation (A. W. Irwin et al. 2004 , in preparation), but a brief discussion of its main characteristics can be found in Cassisi et al. (2003). This EOS can cover the entire stellar structure along all the main evolutionary phases of stars in a large mass range (including the full mass range spanned by our present models); the accuracy of this

\footnotetext{
${ }^{4}$ The EOS code is made publicly available at ftp://astroftp.phys.uvic.ca under the GNU General Public License.
}

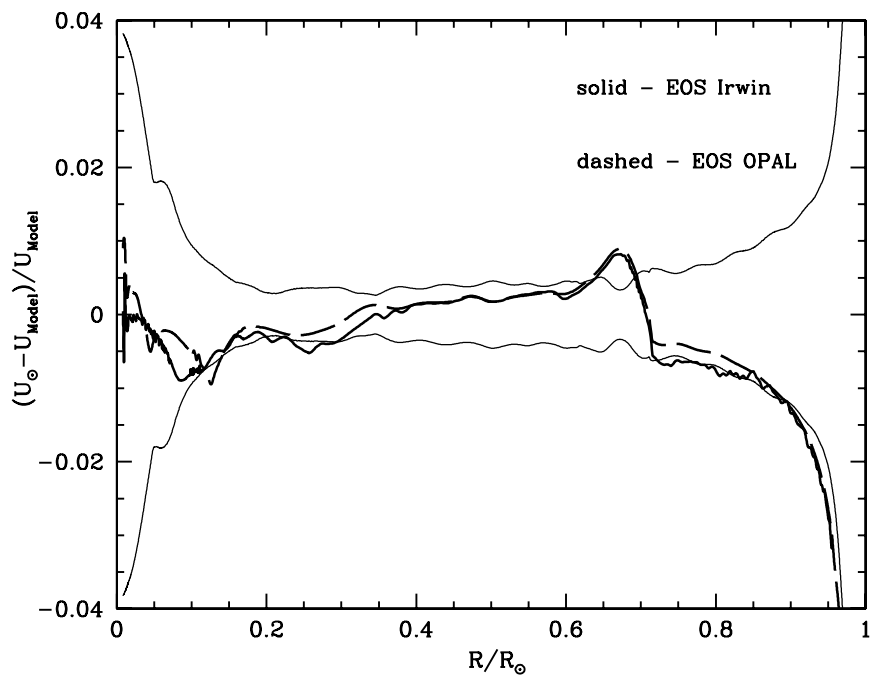

Fig. 1.-Relative differences between the helioseismologically inferred solar $U=P / \rho$ profile (Degl'Innocenti et al. 1997) and the results from two theoretical solar models computed using the OPAL EOS and the Irwin EOS, respectively. Thin solid lines mark the "conservative" error on the helioseismological result as discussed by Degl'Innocenti et al. (1997).

EOS is similar to the case of the OPAL EOS (Rogers et al. 1996) - and recently updated in the treatment of some physical inputs (Rogers \& Nayfonov 2002) - which, however, does not allow the full coverage of the stellar models presented in this work. In fact, for example, the new OPAL EOS still has an upper limit to the temperature of $10^{8} \mathrm{~K}$, and this prevents any evolutionary computation beyond the approach to the $\mathrm{He}$ burning ignition.

To give an idea of the differences between our adopted EOS and the OPAL one in the common range of validity, we show in Figure 1 a comparison of the run of the ratio $U=P / \rho$ as a function of the distance from the star center, obtained from our calibrated solar model (see $\S 4$ ), and a solar model calibrated using the OPAL EOS. In both calibrated models the surface $Z / \mathrm{X}$ ratio, envelope He abundance, and depth of the convective envelope match the empirical determinations (see below and $\S 4)$. In addition to the comparison with the values of $U$ inferred from helioseismology (data from Degl'Innocenti et al. 1997), it is important to note how our adopted EOS closely matches the results obtained with the OPAL EOS.

As for the effect of passing from the EOS used in Cassisi \& Salaris (1997) to the actual one, we performed a test by computing the evolution of a $1 M_{\odot}$ star of solar metallicity up to the He flash, using both EOSs and keeping everything else fixed. With the new Irwin EOS the zero-age main sequence (ZAMS) $\log \left(L / L_{\odot}\right)$ is $\sim 25 \%$ brighter and its effective temperature $\sim 250 \mathrm{~K}$ hotter; the TO $\log \left(L / L_{\odot}\right)$ is $\sim 10 \%$ brighter, its effective temperature $\sim 190 \mathrm{~K}$ higher, and its age is $1.6 \mathrm{Gyr}$ younger ( $\sim 16 \%$ younger). At the He flash this age difference is preserved, whereas the He core mass is $0.01 M_{\odot}$ smaller.

5. Our stellar evolution code can account for the atomic diffusion of both helium and heavy elements. The diffusion coefficients are calculated by means of a routine provided by A. Thoul (1995, private communication), which solves Burgers's equation for a multicomponent fluid (Thoul et al. 1994; see also the in-depth discussion by Schlattl \& Salaris 2003). At variance with canonical computations in which the total metallicity does not change within the star, when diffusion occurs, the metal abundances display a gradient across the stellar structure. However, since all elements heavier than carbon are more or 
less equally diffused, the abundance ratios are not significantly affected by atomic diffusion, and this allows one to account for the effects of diffusion on the stellar opacity simply by interpolating among scaled-solar opacity tables of different $Z$.

We have properly accounted for atomic diffusion only when computing the standard solar models (see $\S 4$ ). The whole set of evolutionary models presented in this paper has been computed without including atomic diffusion. In fact, although in the Sun atomic diffusion is basically fully efficient, spectroscopic observations of, for example, stars in Galactic globular clusters or field halo stars (see, e.g., Gratton et al. 2001; Bonifacio et al. 2002 and references therein) point to a drastically reduced efficiency of diffusion that may be due to the counteracting effect of some sort of rotational mixing processes. This raises the possibility that the almost uninhibited efficiency of diffusion found in the Sun might not be a common occurrence. In view of this uncertainty we decided, conservatively, to not include in our model grid computation the effect of atomic diffusion.

6. The outer boundary conditions have been computed integrating the atmospheric layers with the $T(\tau)$ relation provided by Krishna-Swamy (1966). Superadiabatic convection is treated according to the Cox \& Giuli (1968) formalism of the mixinglength theory (Böhm-Vitense 1958). The mixing-length parameter has been fixed by the solar calibration and kept constant for all masses during all evolutionary phases (see, e.g., Freytag \& Salaris 1999).

7. All models include mass loss using the Reimers (1975) formula with the free parameter $\eta$ set to 0.4 . This value of $\eta$ is commonly assumed (e.g., Girardi et al. 2000) because it allows one to broadly reproduce the mean colors of horizontal branches in Galactic globular clusters. We are in the process of computing an additional set of models (for all metallicities in our grid) that employs $\eta=0.2$, in order to provide a tool to assess the impact of different efficiency of mass loss on various properties of simple and composite stellar populations (e.g., their integrated colors).

\section{THE TREATMENT OF CONVECTION AT THE BORDER OF CONVECTIVE CORE}

Stars with masses larger than $\sim 1.1 M_{\odot}$ (the exact value depends on the initial chemical composition) during the central $\mathrm{H}$-burning phase develop a convective core as a result of the dependence of the CNO cycle efficiency on temperature. The instability against turbulent convection is classically handled by means of the Schwarzschild criterion for a chemically homogeneous fluid. This well-known criterion is based on the comparison between the expected temperature gradient produced by the radiative transport of energy and the adiabatic one. It is worth noting that the proper estimate of the size of the convective region is then primarily dependent on the accuracy of the input physics. Any improvement of the adopted physics may produce a change in the temperature gradients and, in turn, modify the location of the boundaries of the convective regions.

A second important point concerns the possibility that the motion of the convective fluid elements is not halted at the boundary with the stable region beyond the formal convective core. Although beyond the Schwarzschild boundary a moving fluid element is subject to a strong deceleration, it might be possible that a nonzero velocity is maintained along a certain length. This mechanical overshoot can induce a significant amount of mixing in a region formally stable against convection (see Cordier et al. 2002 and references therein). Finally, another important point that has to be taken into account is that a sizeable increase in the internal mixed region, and hence larger convective cores, could be obtained as a consequence of rotationally induced mixing (Meynet \& Maeder 2000).

There are therefore at least three different reasons (inadequate input physics, overshooting from the convective boundary, and additional mixing due to rotation) why the size of the "real" stellar convective cores might be different from the one predicted by "canonical" models, that is, models computed neglecting rotation and overshooting from the Schwarzschild convective boundary. This evidence raises the question whether the size of the convective core, as determined by the (classical) Schwarzschild criterion, is able to properly match the observations or has to be "artificially" increased.

Discrepancies with observations have been usually interpreted in terms of the efficiency of an overshooting mechanism; the extension of this additional mixed region is usually defined in terms of a parameter $\lambda_{\text {ov }}$ that gives the lengthexpressed as a fraction of the local pressure scale height $H_{P}-$ crossed by the convective cells in the convectively stable region outside the Schwarschild convective boundary.

The case for a significant amount of overshooting has been presented many times both theoretically and observationally, although in some cases the results have been contradictory (see, e.g., Testa et al. 1999; Barmina et al. 2002; Brocato et al. 2003). What is well known is the effect of including overshooting in the stellar models: during the core H-burning phase, the star has a larger He core, a brighter luminosity, and a longer lifetime. During the following core He-burning phase, the luminosity is brighter, the lifetime is shorter, and the blue loops in the color magnitude diagram (CMD) are less extended than in the absence of overshooting. Theory therefore predicts that the mass-luminosity $(M-L)$ relationship for stars crossing the Cepheid instability strip is largely affected by the amount of overshooting accounted for in the stellar evolution computations. The comparison of the theoretical $M-L$ relationship with empirical data could, in principle, put tight constraints on the efficiency of this process. This approach has been recently adopted by Keller \& Wood (2002) by adopting a large database of empirical data for bump Cepheids, and the obtained results seem to support the occurrence of a large overshooting efficiency. Although the method is sound, the results have to be cautiously treated, as clearly shown by Cassisi (2004).

Because of the lack of a general consensus about the convective core overshooting efficiency, our stellar models are computed both without overshooting and with a significant efficiency of this process. In the latter case, we adopt $\lambda_{\mathrm{ov}}=$ $0.20 H_{P}$. This value allows a good match between the CMD of Galactic open clusters of different ages and allows us a comparison with the evolutionary models recently presented by other groups, all using a similar value of the overshooting parameter. The overshooting region is fully mixed as far as the chemical elements are concerned, and the temperature gradient is kept at its radiative value.

Another important issue to be addressed is the value of $\lambda_{\mathrm{ov}}$ when stars have small convective cores. It is well known that the TO morphology of the evolutionary tracks and, in turn, of the isochrones, does depend on how the extent of the convective core decreases with decreasing mass. When moving deeper and deeper inside the star, the pressure scale height steadily increases; this causes a large increase of the size of convective cores in stars whose Schwarzschild convective boundary is fast shrinking (e.g., for masses below $\approx 1.5 M_{\odot}$ ) if the overshooting efficiency is kept fixed at a constant fraction 


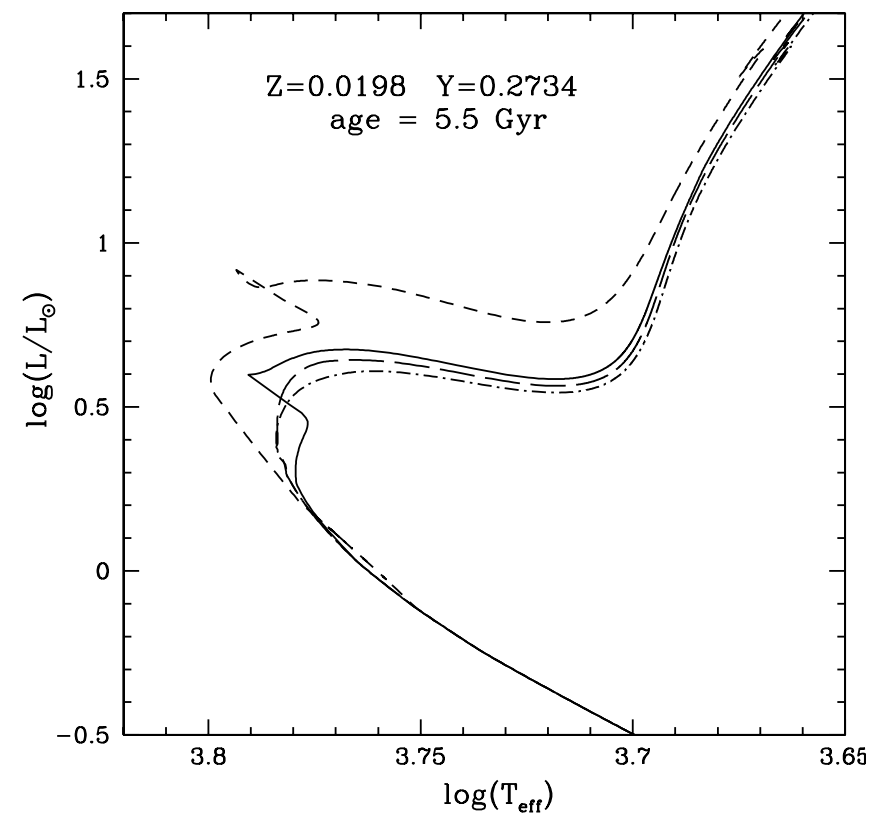

FIG. 2.-Theoretical isochrones with the same (see labels) age and chemical composition, computed with different ways to decrease the core overshoot efficiency with stellar mass: the dot-dashed line corresponds to canonical models; the solid line has been obtained from models computed using the prescription adopted in this work (see text for more details); the short-dashed line has been obtained by assuming that $\lambda_{\mathrm{ov}}$ is equal to $0.20 H_{P}$ for stellar masses larger than $1.3 M_{\odot}$, equal to 0 for $M \leq 0.95 M_{\odot}$, and linearly decreasing in the intermediate range of masses; the long-dashed line has been obtained by assuming that $\lambda_{\mathrm{ov}}$ is equal to $0.20 H_{P}$ for stellar masses larger than $1.7 M_{\odot}$, equal to $0.15 H_{P}$ for $1.5 M_{\odot}$, vanishing for $1.1 M_{\odot}$, and linearly decreasing in the intermediate range.

of $H_{P}$. It is clear that we must decrease $\lambda_{\text {ov }}$ to zero for stars with small convective cores, a problem addressed theoretically, for example, by Roxburgh (1992) and Woo \& Demarque (2001).

In order to show how critical this issue is, we show in Figure 2 two isochrones of the same chemical composition and age, obtained using different assumptions about the trend of $\lambda_{\mathrm{ov}}$ with mass. The change in the isochrone morphology is quite significant, and different choices concerning the core overshoot efficiency in the critical mass range $1.0 \geq M / M_{\odot} \leq 1.5^{5}$ mimic different isochrone ages. It is also worthwhile to note the significant change in the shape of the TO region. This means that the trend of $\lambda_{\text {ov }}$ with mass, for masses with small convective cores, potentially introduces an additional degree of freedom in stellar evolution models. Even if this problem affects only a restricted range of cluster ages, it has to be taken into account when discussing the uncertainties affecting stellar models.

In our models with core overshooting, regardless of the initial metallicity, we have chosen the following algorithm for varying the overshoot efficiency with mass: for masses larger or equal to $1.7 M_{\odot}$, we fixed $\lambda_{\mathrm{ov}}$ at $0.20 H_{P}$; for stars less massive than $1.1 M_{\odot}$ we use $\lambda_{\text {ov }}=0$ (we checked that all our models of at least $1.1 M_{\odot}$ show a central convective core during the MS), while in the intermediate range $\lambda_{\mathrm{ov}}$ varies according to the relation $\lambda_{\mathrm{ov}}=\left(M / M_{\odot}-0.9\right) / 4$. We have verified by performing several numerical experiments that this choice allows a smooth variation of the isochrone TO

\footnotetext{
${ }^{5}$ It is worth noting that the finer details of the isochrone morphology do depend not only on the adopted overshoot efficiency, but also on the number of evolutionary tracks used for computing the isochrones.
}

morphology and a smooth decrease to zero of the convective cores for stars in this mass range.

Moreover, this choice for the overshooting extension does provide a good fit to the TO morphology of a sample of clusters of various ages, as discussed later. In particular, our overshooting isochrones fit extremely well the TO morphology of clusters like NGC 2420 and NGC 6819, where the typical TO mass is $\sim 1.3 M_{\odot}$ (for $\left.[\mathrm{Fe} / \mathrm{H}]=-0.44\right)$ and $\sim 1.4 M_{\odot}$ (for $[\mathrm{Fe} / \mathrm{H}]=0.06)$, respectively, i.e., in a mass range where the $\lambda_{\mathrm{ov}}$ is decreasing according to our prescription. The case of M67 (Sandquist 2004; see also below) and the eclipsing binary AI Phe (see below) seem to indicate, however, that $\lambda_{\mathrm{ov}} \sim 0$ when the stellar mass is equal to $\sim 1.2 M_{\odot}$. Since we provide both overshooting and canonical models, the user can freely choose to use a criterion based on age for the switch to $\lambda_{\mathrm{ov}}=0$ models and isochrones.

As for the convective cores during the He-burning phase, we account for semiconvection (which is driven by mechanical overshooting from the boundary of the Schwarzschild core) at the border of the canonical convective core by using the numerical scheme described by Castellani et al. (1985). Near the core helium exhaustion we inhibited the breathing pulses (Castellani et al. 1985; Dorman \& Rood 1993), following the results of Cassisi et al. (2003). The breathing pulses are suppressed, adopting the procedure suggested by Caputo et al. (1989), i.e., by limiting the extent of the convective core so that the central helium abundance cannot increase between consecutive models.

We neglect the possible occurrence of overshooting from the bottom of the convective envelopes. This choice has been made in order to reduce as much as possible the number of free parameters in the stellar computations and also in light of the fact that there is no clear-cut empirical evidence for a sizable efficiency of this phenomenon. A critical test for this phenomenon is the comparison between the position of the RGB bump in Galactic globular clusters with the theoretical counterpart. A nonnegligible amount of overshooting from the bottom of the convective envelope does shift the bump to lower luminosities. This kind of test does not provide a definitive answer (e.g., Riello et al. 2003), mainly because of the uncertainty on the metallicity scale of globular clusters (the bump position is also strongly affected by the initial stellar metallicity). It is, however, important to remark that convective envelope overshooting can affect the morphology of blue loops during the core He-burning phase (Alongi et al. 1991; Renzini et al. 1992; Renzini \& Ritossa 1994). A detailed investigation of the dependence of blue loops on the physical assumptions adopted in stellar computations and a comparison with Cepheid data will be the subject of a forthcoming paper.

\section{THE STANDARD SOLAR MODEL}

The calibration of the standard solar model allows one to fix the value of the mixing length, as well as the solar initial $\mathrm{He}$ and metal fractions. In practice, one finds the combination of initial $\mathrm{He}\left(Y_{\odot}\right)$ and metal $\left(Z_{\odot}\right)$ mass fractions, and the value of the mixing-length parameter that reproduce the solar radius $\left(R_{\odot}=6.960 \times 10^{10} \mathrm{~cm}\right)$, luminosity $\left(L_{\odot}=3.842 \times\right.$ $10^{33}$ ergs $\mathrm{s}^{-1}$; Bahcall \& Pinsonneault 1995), and ratio $(Z / X)=0.0245 \pm 0.005$ (Grevesse \& Noels 1993), at an age of $t_{\odot}=4.57 \mathrm{Gyr}$.

The accuracy of the theoretical solar model can be then tested by comparing the depth of the outer convective layers and the surface $Y$-value with the values $0.710-0.716 R_{\odot}$ 
(Castellani et al. 1997) and 0.2438-0.2443 (Dziembowski et al. 1995), respectively, as obtained from helioseismological studies.

It is well known (see, e.g., Bahcall \& Pinsonneault 1992, 1995; Basu et al. 1996; Bahcall et al. 2001 and references therein) that solar models without microscopic diffusion cannot properly account for some features of the seismic Sun. For this reason, we decided to account for atomic diffusion of both helium and metals when computing solar models, as described in $\S 2$.

We have computed several models with mass equal to $1 M_{\odot}$ starting from the pre-MS phase, under different assumptions about the initial $\mathrm{He}$ and metal abundances and mixing-length parameter. The "best" solar model that fulfills the quoted requirements has been obtained for an initial $Y_{\odot}=0.2734$, an initial $Z_{\odot}=0.0198$, and a mixing-length value equal to 1.913 . This standard solar model is in agreement with the results from helioseismology concerning the depth of the convective envelope $\left(0.716 R_{\odot}\right)$ and the present surface He abundance $(Y=$ $0.244)$, and with the actual $(Z / X)$ ratio $[(Z / X)=0.0244]$. The value of the mixing length obtained from the calibration of the solar standard model is then adopted for the whole set of models presented in this work. We show later that this choice of the mixing length coupled with the color-effective temperature transformations used in this work allows a fine match to several observational constraints. The initial solar He abundance and metallicity $\left(Y_{\odot}=0.2734-Z_{\odot}=0.0198\right)$ are the values adopted when computing the so-called solar metallicity model set.

\section{THE STELLAR MODEL LIBRARY}

\subsection{The Initial Chemical Composition of the Model Grid}

As already stated, this work provides a homogeneous and self-consistent database of theoretical stellar models and isochrones, based on the scaled solar heavy-element distribution by Grevesse \& Noels (1993). The extension to models accounting for a suitable enhancement of $\alpha$-elements will be the subject of a forthcoming paper.

In order to cover a wide range of chemical compositions, we provide models computed for 10 different metallicities, namely, $Z=0.0001,0.0003,0.001,0.002,0.004,0.008,0.01$, $0.0198,0.03$, and 0.04 . As far as the initial He-abundances are concerned, we adopt for the lowest metallicity the estimate recently provided by Cassisi et al. (2003; see also Salaris et al. 2004) based on new measurements of the $R$ parameter in a large sample of Galactic globular clusters. They found an initial He abundance for globular cluster stars on the order of $Y=0.245$, which is in satisfactory agreement with the recent determination of the cosmological baryon density provided by Wilkinson Microwave Anisotropy Probe observations of the power spectrum of the cosmic microwave background (CMB) radiation (Spergel et al. 2003). It is worth noting that the theoretical $R$ parameter calibration used by Cassisi et al. (2003) is based on a small set of low-mass $\alpha$-enhanced stellar models computed with exactly the same code and input physics of the models presented here. In order to reproduce our initial $Y_{\odot}($ see $\S 4)$, we adopt $d Y / d Z \sim 1.4$.

In Table 1 we summarize the initial chemical composition of our model grid and also provide the corresponding $[\mathrm{Fe} / \mathrm{H}]$ value (determined assuming the observed solar $(Z / X)$ ratio by Grevesse \& Noels 1993), a quantity directly comparable to spectroscopic observations. It is important to note that the solar metallicity models provide $[\mathrm{Fe} / \mathrm{H}]=0.06$, instead of 0 . The
TABLE 1

Initial Chemical Compositions of Our Model Grid

\begin{tabular}{|c|c|c|}
\hline$Z$ & $Y$ & {$[\mathrm{Fe} / \mathrm{H}]$} \\
\hline $0.0001 \ldots .$. & 0.245 & -2.27 \\
\hline $0.0003 \ldots$ & 0.245 & -1.79 \\
\hline $0.0010 \ldots$. & 0.246 & -1.27 \\
\hline $0.0020 \ldots \ldots \ldots \ldots$ & 0.248 & -0.96 \\
\hline 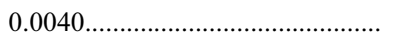 & 0.251 & -0.66 \\
\hline $0.0080 \ldots \ldots \ldots \ldots$ & 0.256 & -0.35 \\
\hline 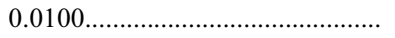 & 0.259 & -0.25 \\
\hline $0.0198 \ldots \ldots \ldots \ldots$ & 0.273 & 0.06 \\
\hline $0.0300 \ldots \ldots \ldots \ldots$ & 0.288 & 0.26 \\
\hline $0.0400 \ldots$. & 0.303 & 0.40 \\
\hline
\end{tabular}

reason is that the model grid does not include diffusion (which we know is active in the Sun but, as discussed before, is possibly inhibited, at least at the surface of other low-mass stars); when diffusion is included, the solar metallicity composition would provide $[\mathrm{Fe} / \mathrm{H}]=0$ only at the solar age for solar-like models. This also means that our model and isochrone grid computed with the solar initial chemical composition will be unable to match the properties of the Sun, for example, the helioseismologically determined depth of the convective envelope and the envelope $\mathrm{He}$ abundance, the surface $\mathrm{Z} / \mathrm{X}$ ratio, and in general the inner sound speed profile. This is common to all databases computed without diffusion, but on the other hand our same solar isochrones are able to fit-as we show laterthe MS (and the rest of the observed CMD) of the open cluster M67, whose stars display a well-established solar photospheric $[\mathrm{Fe} / \mathrm{H}]$, has an age close to the solar value, and whose reddening and distance are well determined empirically.

\subsection{The Evolutionary Tracks and Isochrones}

For each chemical composition, we have computed the evolution of up to 41 different stellar masses. The minimum mass is around $0.50 M_{\odot}$ (slightly varying with metallicity), while the maximum mass is always equal to $10 M_{\odot}$. In the near future we will extend the computations to both very low mass $\operatorname{stars}^{6}$ and more massive stars.

In order to increase the accuracy of the numerical interpolations among the different evolutionary tracks needed for computing isochrones and for population synthesis studies, and to allow a detailed analysis of specific evolutionary properties, such as the RGB transition (Sweigart et al. 1990), we adopted a mass step of $0.1 M_{\odot}$ (or lower) for masses below $2.5 M_{\odot}, 0.2 M_{\odot}$ for masses in the range $2.5 \leq M / M_{\odot} \leq 3.0$, and $0.5 M_{\odot}$ for more massive models. ${ }^{7}$

Models less massive than $\sim 3 M_{\odot}$ have been computed starting from the pre-MS phase, whereas the more massive ones have been computed starting from a chemically homogeneous configuration on the MS. All models, apart from the less massive ones whose central H-burning evolutionary times are longer than the Hubble time, have been evolved until the $\mathrm{C}$ ignition or after the first few thermal pulses along the asymptotic giant branch (AGB) phase. We plan in the near future to extend the evolution of low- and intermediate-mass

\footnotetext{
${ }^{6}$ This work will be done in collaboration with A. W. Irvin and D. Vandenberg.

The mass grid is slightly more coarse for the model grid accounting for core overshooting.
} 
stars up to the end of the thermal pulses along the AGB, using the synthetic AGB technique (see, e.g., Marigo et al. 1996 and reference therein).

In the case of models undergoing a violent He flash at the RGB tip, we do not perform a detailed numerical computation of this phase; instead, we consider the evolutionary values of both the He core mass $\left(M_{c \mathrm{He}}\right)$ and the surface He abundance $\left(\mathrm{He}_{S}\right)$ at the RGB tip and accrete the envelope with the evolutionary chemical composition until the appropriate total mass is obtained. After waiting for the thermal relaxation of the structure, the resulting zero-age horizontal branch (ZAHB) model has the same luminosity of the corresponding model evolved through the He flash, as demonstrated by Vandenberg et al. (2000).

A subsample of the evolutionary tracks (canonical models) is shown in Figure 3 for all our adopted chemical compositions. It is evident that all relevant evolutionary phases are properly sampled by the different tracks.

For each chemical composition we have computed additional He burning models (that is, $\mathrm{HB}$ models in addition to the ones obtained with our chosen mass-loss law) with various different values of the total stellar mass after the RGB phase, using a fixed core mass and envelope chemical profile, as given by a RGB progenitor having an age (at the RGB tip) of $\sim 13$ Gyr (see Fig. 4). These models allow one to compute the CMD of synthetic HB populations, where there is a spread in the amount of mass lost along the RGB phase (as in the case of Galactic globular clusters).

The RGB progenitor of these additional HB models has a mass typically equal to $0.8 M_{\odot}$ at the lowest metallicities, and increasing up to $1.0 M_{\odot}$ for the more metal-rich compositions. For these additional computations we have adopted a very fine mass spacing; i.e., more than $30 \mathrm{HB}$ models have been computed for each chemical composition. A subset of these models in displayed in Figure 4. The very fine mass spacing provides a suitable tool for investigating the pulsational properties of RR Lyrae stars and Population II Cepheids as a function of the cluster metallicity and HB morphology.

All our post-ZAHB computations have been extended either to the onset of thermal pulses for more massive models or until the luminosity along the cooling sequence of white dwarfs decreases down to $\log \left(L / L_{\odot}\right) \sim-2.5$ for less massive models. They allow a detailed investigation of the various evolutionary paths following the exhaustion of the central He burning, i.e., (1) extremely hot horizontal branch stars that do not reach the AGB and evolve as AGB-manqué stars, (2) post early AGB stars, which leave the AGB before the onset of thermal pulses, and (3) bona fide AGB stars, i.e., stars massive enough to experience the AGB thermal pulses.

In order to produce isochrones for a given initial chemical composition, the individual evolutionary tracks have been reduced to the same number of points, according to a wellestablished procedure (see, e.g., Prather 1976; Bergbusch \& Vandenberg 1992). Along each evolutionary track some characteristic homologous points (key points [KPs]) corresponding to well-defined evolutionary phases have been identified. The choice and number of KPs had to fulfill two conditions: (1) all main evolutionary phases have to be properly accounted for, and (2) the number of KPs has to be large enough to allow a suitable sampling of the track morphology, even for faster evolutionary phases. The second condition requires also that the number of points between two consecutive KPs has to be properly chosen.

As is well known, when passing from stars with a radiative core during the central H-burning phase to more massive stars with convective cores, the track morphology changes significantly. Therefore, the KPs in this phase differ for stars with radiative or convective cores. The adopted KPs and the number of points between consecutive KPs are listed in Table 2, while in Figure 5 we show the location of the KPs along a typical intermediate-mass star and a low-mass He-burning structure. This choice allows an adequate sampling of the track morphology for the whole evolutionary database, and it guarantees accurate interpolations when using this database in population synthesis codes.

Since we have exactly the same KPs at all metallicities and the same number of points distributed between consecutive KPs, all isochrones at any age and metallicity will have the same number of points distributed consistently between corresponding evolutionary phases. This allows one to easily produce isochrones of intermediate metallicities by simply interpolating on a point-by-point basis among the available isochrones. In this way, the use of our database in a stellar population synthesis algorithm is straightforward and simple. To that end, we have performed a numerical test as follows. Isochrones for various ages and $Z=0.008([\mathrm{Fe} / \mathrm{H}]=-0.35)$ have been computed by interpolating (on a point-by-point basis) quadratically among the chemical compositions corresponding to $Z=0.004([\mathrm{Fe} / \mathrm{H}]=-0.66), Z=0.01([\mathrm{Fe} / \mathrm{H}]=$ $-0.25)$, and $Z=0.0198([\mathrm{Fe} / \mathrm{H}]=0.06)$. These isochrones have been compared to the actual isochrones of the same metallicity $(Z=0.008$; i.e., $[\mathrm{Fe} / \mathrm{H}]=-0.35)$, computed from the appropriate evolutionary tracks. We found that a simple quadratic interpolation scheme is sufficient to reproduce the evolving masses to within a few thousandths of solar masses, colors, and magnitudes within 0.02 mag or better along most of the evolutionary phases spanned by the isochrones, $\log \left(L / L_{\odot}\right)$ and $\log \left(T_{\text {eff }}\right)$, within a few thousandths of a dex along the isochrones.

The whole set of evolutionary computations (but the additional HB models discussed above) have been used to compute isochrones for a large age range, namely, from $40 \mathrm{Myr}$ up to 12.5 Gyr (older isochrones can also be computed), from the ZAMS up to the first thermal pulse on the AGB or to the $\mathrm{C}$ ignition. In the near future, we will make available a World Wide Web interface ${ }^{8}$ that will allow any user to compute isochrones, evolutionary tracks, and synthetic CMD (see below) for any specified age, stellar mass, star formation history, and so on (D. Cordier et al. 2004, in preparation).

Detailed tables displaying the relevant evolutionary features for all computed tracks are also available via ftp and at the Web site. ${ }^{9}$ We display in Tables 3 and 4 a summary of the basic information about the $[\mathrm{Fe} / \mathrm{H}]=0.06$ canonical models in selected evolutionary phases; tables like these, for all metallicities and both canonical and overshooting models, are available at the aforementioned Web site.

The files containing the isochrones provide at each point (2000 points in total) the initial value of the evolving mass and the actual value (in principle different, due to the effect of mass loss $), \log \left(L / L_{\odot}\right), \log \left(T_{\text {eff }}\right), M_{V},(U-B),(B-V),(V-I)$, $(V-R),(V-J),(V-K),(V-L)$, and $(H-K)$ on the Johnson-Cousins system. The transformation from $\log \left(L / L_{\odot}\right)$

\footnotetext{
${ }^{8}$ The entire library of evolutionary tracks and isochrones are available on request to one of the authors or can be retrieved at http://www.te.astro.it/ BASTI/index.php.

9 Those interested in obtaining more information, specific evolutionary results, as well as additional set of models, can contact directly one of the authors or use the request form available at the quoted Web site.
} 


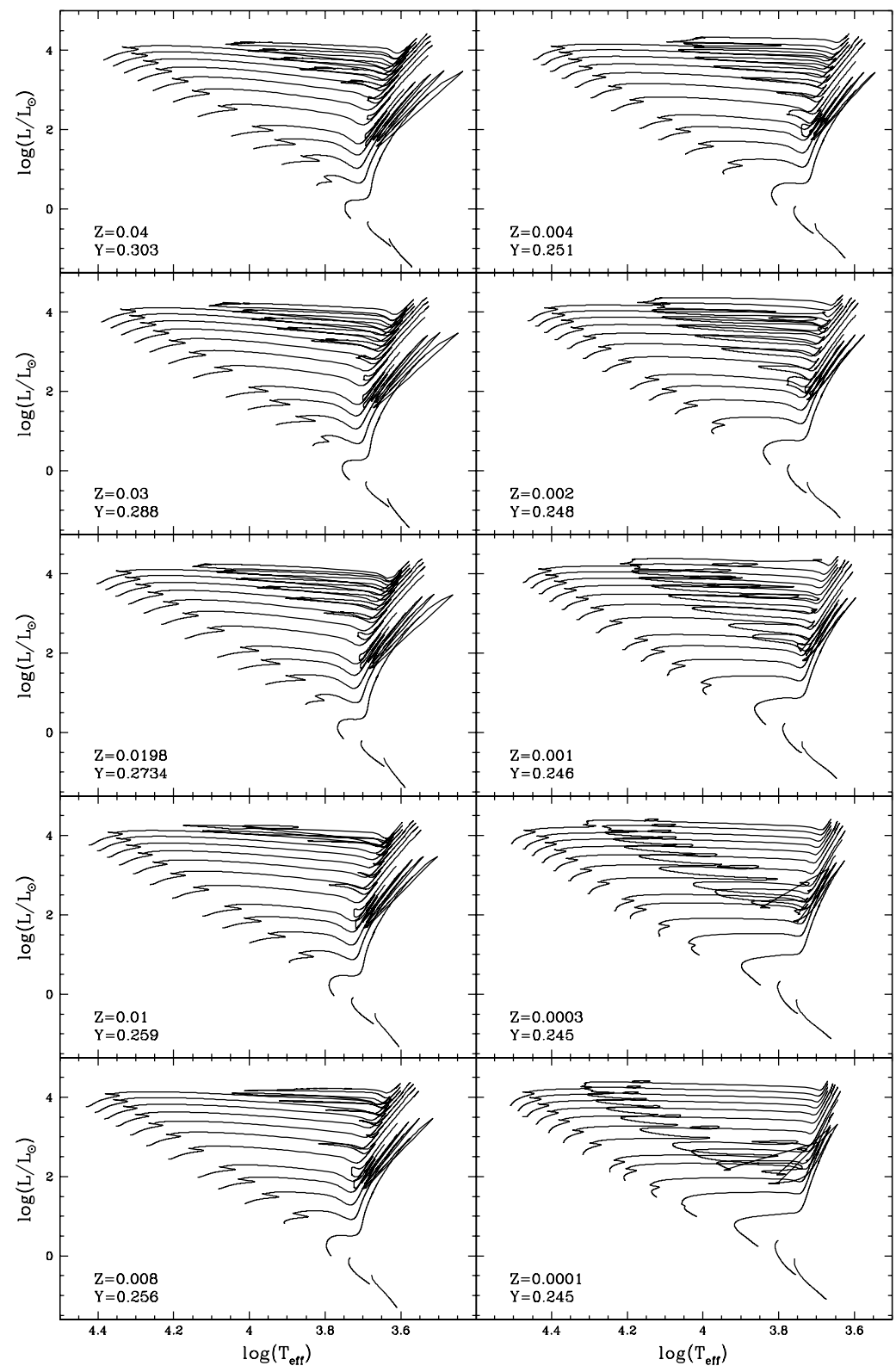

FIG. 3.-Selected evolutionary tracks for the 10 metallicities of our model grid. The plotted tracks correspond to models with the following initial masses: $M / M_{\odot}=0.5,0.7,1.0,1.5,2.0,2.5,3.0,4.0,5.0,6.0,7.0,8.0,9.0$, and 10.0 . 


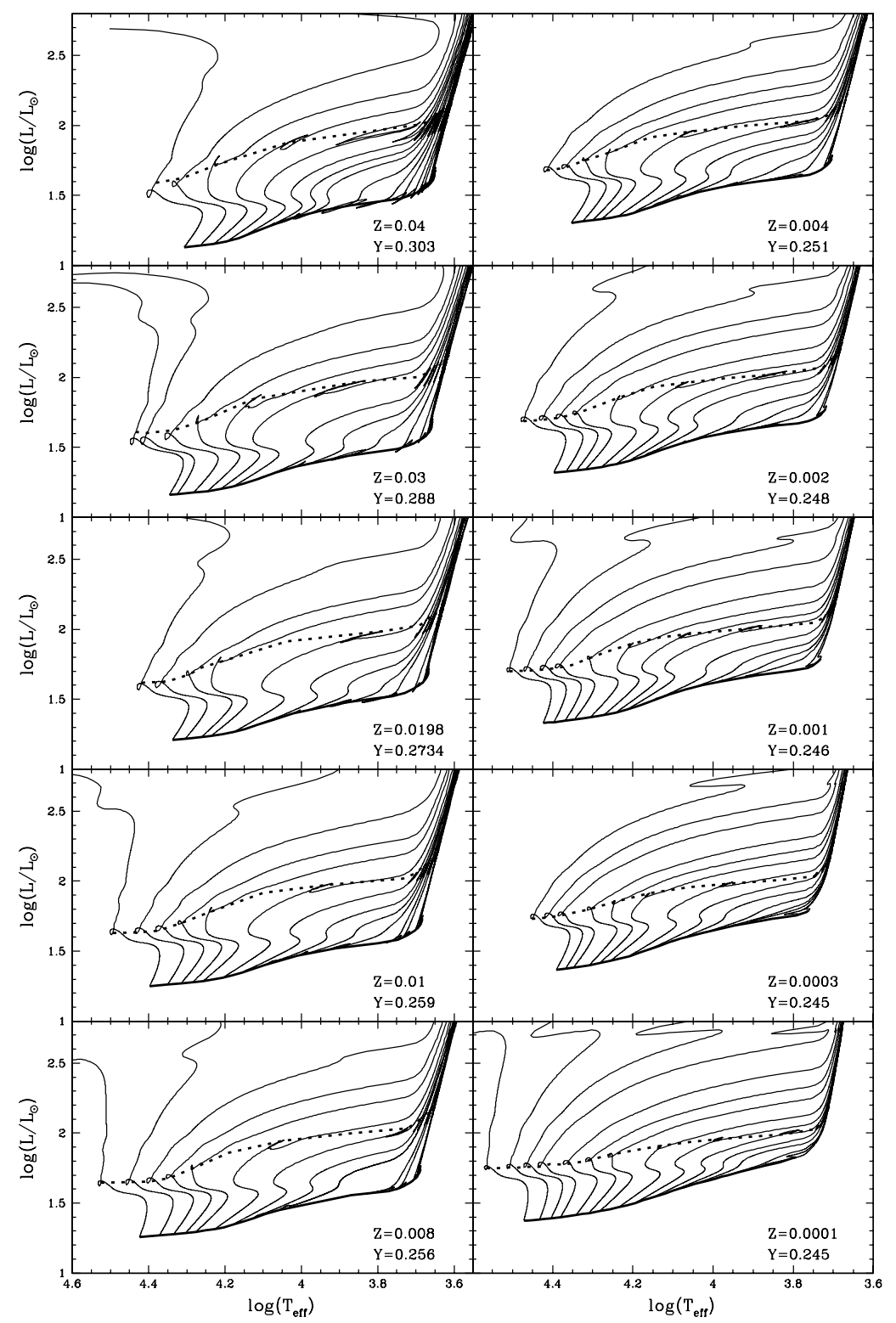

FIG. 4.-Additional HB evolutionary tracks suited for the computation of synthetic HB populations. The figure also shows the location of the ZAHB and central He exhaustion loci. 
TABLE 2

The Adopted KPs along the Evolutionary Tracks and the Number of Normalized Points between Consecutive KPs

\begin{tabular}{|c|c|c|}
\hline KP & Evolutionary Phase & Number of Normalized Points \\
\hline $1 \ldots \ldots \ldots \ldots . . . .$. & Start of the central H-burning phase & 1 \\
\hline 2 & $1^{\circ}$ minimum in $T_{\text {eff }}$ for $\mathrm{HM}$ or $X_{c}=0.30$ for LM & 200 \\
\hline 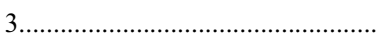 & Maximum in $T_{\text {eff }}$ along the MS & 60 \\
\hline 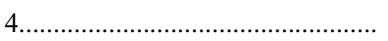 & Maximum in luminosity for $\mathrm{HM}$ or $X_{c}=0.0$ for LM stars & 60 \\
\hline 5 & Minimum in luminosity for HM or base of the RGB for LM & 70 \\
\hline 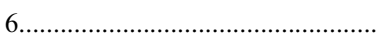 & Tip of the RGB & 800 \\
\hline 7 & Start of the quiescent central He-burning phase & 10 \\
\hline 8 & Central abundance of $\mathrm{He}$ equal to 0.55 & 150 \\
\hline 9 & Central abundance of He equal to 0.50 & 100 \\
\hline $10 \ldots \ldots \ldots-1$ & Central abundance of He equal to 0.40 & 100 \\
\hline 11 & Central abundance of $\mathrm{He}$ equal to 0.20 & 80 \\
\hline $12 \ldots \ldots \ldots \ldots \ldots \ldots$ & Central abundance of $\mathrm{He}$ equal to 0.10 & 80 \\
\hline $13 \ldots \ldots \ldots \ldots$ & Central abundance of He equal to 0.00 & 140 \\
\hline 14 & $\begin{array}{l}L_{\mathrm{CNO}}>L_{3 \alpha} \text { during the } \mathrm{AGB} \text { phase } \\
\text { (Carbon ignition for stars with nondegenerate } \mathrm{CO} \text { cores) }\end{array}$ & 150 \\
\hline \multicolumn{3}{|c|}{ Horizontal Branch Models } \\
\hline 1 & Start of the central He-burning phase & 1 \\
\hline 2 & Central abundance of He equal to 0.50 & 130 \\
\hline 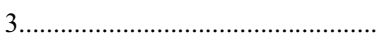 & Central abundance of He equal to 0.40 & 50 \\
\hline 4 & Central abundance of He equal to 0.20 & 120 \\
\hline 5 & Central abundance of He equal to 0.10 & 70 \\
\hline 6. & Central abundance of He equal to 0.00 & 80 \\
\hline 7 & Maximum in luminosity along the clump on the AGB & 150 \\
\hline 8 & Minimum in luminosity along the clump on the AGB & 50 \\
\hline 9 & Maximum in luminosity all along the track & 250 \\
\hline
\end{tabular}

Note.-HM denotes MS Stars with convective cores; LM denotes stars with radiative cores on the MS.

and $\log \left(T_{\text {eff }}\right)$ to magnitudes and colors has been performed adopting a new set of bolometric corrections and color- $T_{\text {eff }}$ transformations specifically computed for this work (see $\S 5.3$ for more details).

These isochrones can be used as input data for the fortran code SYNTHETIC MAN(ager) that we have written in order to
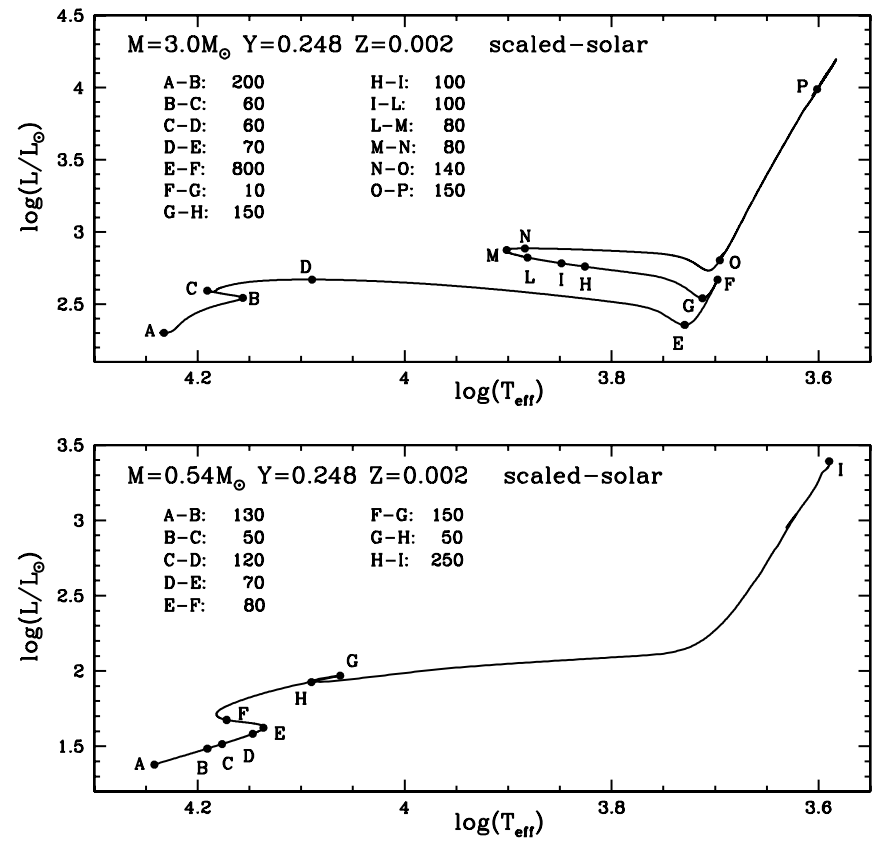

FIG. 5.-Location of the KPs along selected evolutionary tracks. compute synthetic CMDs, integrated colors, and integrated magnitudes of a generic stellar population with an arbitrarily chosen star formation rate (SFR) and age metallicity relation (AMR). The program reads a grid of isochrones for ages between $\log t=7.6$ and 10.10 (age $t$ in years), in steps of $0.05 \mathrm{dex}$, and a file specifying an SFR and AMR. For each time step set by the ages of the input isochrones, the program determines from the SFR and AMR the number of stars formed at that age and their metallicity. Once these quantities are computed, the program selects values for the masses of the stars formed, according to a prescribed initial mass function (IMF), and interpolates among the isochrones in the grid (linear interpolation in mass, quadratic interpolation in metallicity) in order to determine the photometric properties for these objects.

The program can also account for a spread in the AMR, photometric errors, depth effects, reddening, and unresolved binaries. In Figure 6 we show an example of CMD for a synthetic population produced by this program. The simulation contains $\sim 90,000$ stars, for a star formation history typical of the solar neighborhood (Rocha-Pinto et al. 2000a, 2000b), using a Salpeter IMF, a $1 \sigma$ photometric error of $0.03 \mathrm{mag}$, and a $20 \%$ fraction of unresolved binaries (the mass of the secondary stars in these systems is determined following Woo et al. 2003). ${ }^{10}$

\subsection{Color Transformations and Bolometric Corrections}

The UBVRIJHKL synthetic photometry was computed with passbands, zero points, and bolometric corrections as

${ }^{10}$ In the near future, any user will be enable to run this program for generating synthetic CMDs by using a Web interface at our Web site. 
TABLE 3

Selected Evolutionary and Structural Properties for Low- and Intermediate-Mass Models with $[\mathrm{Fe} / \mathrm{H}]=0.06$ (No Overshooting)

\begin{tabular}{|c|c|c|c|c|c|c|c|c|c|c|c|c|c|c|c|c|c|c|c|}
\hline$M^{\mathrm{a}}$ & $\log L^{\mathrm{b}}$ & $\log T_{e}^{\mathrm{c}}$ & $M_{\mathrm{cc}}{ }^{\mathrm{d}}$ & $M_{\mathrm{He}}^{\mathrm{e}}$ & $t_{H}^{\mathrm{f}}$ & $\log L^{\mathrm{g}}$ & $\log T_{e}^{\mathrm{h}}$ & $\mathrm{He}_{s}{ }^{\mathrm{i}}$ & $\operatorname{Age}^{j}$ & $M_{\mathrm{He}}{ }^{\mathrm{k}}$ & $M_{\mathrm{cc}}{ }^{1}$ & $M_{\mathrm{He}}^{\mathrm{m}}$ & $M_{\mathrm{CO}}{ }^{\mathrm{n}}$ & $t_{\mathrm{He}}{ }^{\circ}$ & $M_{\mathrm{CO}}^{\mathrm{p}}$ & $M_{\mathrm{He}}^{\mathrm{q}}$ & $\log L^{\mathrm{r}}$ & $\log T_{e}^{\mathrm{s}}$ & $\mathrm{Age}^{\mathrm{t}}$ \\
\hline $0.95 \ldots \ldots \ldots \ldots$ & -0.258 & 3.738 & 0.034 & 0.1092 & 14052.1 & 3.472 & 3.464 & 0.295 & 15301.2 & 0.4782 & 0.115 & 0.4944 & 0.2116 & 95.4 & 0.4767 & 0.5104 & 3.245 & 3.523 & 15413.2 \\
\hline $1.00 \ldots \ldots \ldots \ldots$ & -.161 & 3.751 & 0.053 & 0.1110 & 11513.3 & 3.475 & 3.463 & 0.296 & 12644.1 & 0.4777 & 0.114 & 0.4993 & 0.2168 & 95.0 & 0.4831 & 0.5156 & 3.310 & 3.502 & 12754.6 \\
\hline $1.10 \ldots \ldots \ldots \ldots$ & 0.018 & 3.771 & 0.084 & 0.1177 & 7913.1 & 3.479 & 3.479 & 0.297 & 8869.1 & 0.4775 & 0.112 & 0.5046 & 0.1910 & 94.1 & 0.4885 & 0.5201 & 3.293 & 3.509 & 8977.6 \\
\hline $1.20 \ldots \ldots \ldots \ldots$ & 0.213 & 3.790 & 0.066 & 0.1224 & 5640.7 & 3.482 & 3.489 & 0.295 & 6482.0 & 0.4774 & 0.113 & 0.5075 & 0.1825 & 92.6 & 0.4911 & 0.5220 & 3.319 & 3.513 & 6589.3 \\
\hline $1.30 \ldots \ldots \ldots \ldots$ & 0.400 & 3.813 & 0.015 & 0.1319 & 4138.6 & 3.484 & 3.498 & 0.293 & 4880.8 & 0.4776 & 0.114 & 0.5103 & 0.2057 & 92.0 & 0.4938 & 0.5244 & 3.335 & 3.517 & 4991.7 \\
\hline $1.40 \ldots \ldots \ldots \ldots$ & 0.556 & 3.831 & 0.083 & 0.1401 & 3043.3 & 3.484 & 3.507 & 0.291 & 3821.2 & 0.4777 & 0.117 & 0.5123 & 0.2058 & 91.2 & 0.4977 & 0.5283 & 3.488 & 3.506 & 3926.6 \\
\hline $1.50 \ldots \ldots \ldots \ldots$ & 0.691 & 3.851 & 0.125 & 0.1459 & 2312.5 & 3.484 & 3.513 & 0.289 & 3032.7 & 0.4778 & 0.113 & 0.5139 & 0.2058 & 90.9 & 0.4980 & 0.5277 & 3.346 & 3.532 & 3137.5 \\
\hline $1.60 \ldots \ldots \ldots \ldots .$. & 0.812 & 3.874 & 0.159 & 0.1624 & 1844.3 & 3.485 & 3.520 & 0.288 & 2433.8 & 0.4776 & 0.112 & 0.5161 & 0.2057 & 90.2 & 0.4990 & 0.5289 & 3.339 & 3.535 & 2538.0 \\
\hline $1.70 \ldots \ldots \ldots \ldots$ & 0.923 & 3.897 & 0.194 & 0.1706 & 1520.8 & 3.483 & 3.524 & 0.286 & 1965.5 & 0.4774 & 0.114 & 0.5171 & 0.2059 & 89.9 & 0.5016 & 0.5308 & 3.390 & 3.535 & 2069.4 \\
\hline $1.80 \ldots \ldots \ldots \ldots$ & 1.019 & 3.919 & 0.226 & 0.1803 & 1333.3 & 3.475 & 3.530 & 0.285 & 1599.5 & 0.4760 & 0.119 & 0.5182 & 0.2059 & 90.7 & 0.5019 & 0.5310 & 3.369 & 3.543 & 1703.8 \\
\hline $1.90 \ldots \ldots \ldots \ldots$ & 1.114 & 3.939 & 0.256 & 0.1905 & 1142.9 & 3.458 & 3.536 & 0.284 & 1320.3 & 0.4726 & 0.111 & 0.5167 & 0.2111 & 92.6 & 0.4997 & 0.5297 & 3.339 & 3.550 & 1426.5 \\
\hline $2.00 \ldots \ldots \ldots \ldots$ & 1.207 & 3.956 & 0.292 & 0.2016 & 946.5 & 3.420 & 3.544 & 0.283 & 1106.9 & 0.4652 & 0.109 & 0.5132 & 0.2115 & 97.1 & 0.4961 & 0.5265 & 3.353 & 3.553 & 1217.7 \\
\hline $2.10 \ldots \ldots \ldots \ldots$ & 1.291 & 3.973 & 0.320 & 0.2106 & 836.0 & 3.364 & 3.553 & 0.283 & 943.4 & 0.4546 & 0.113 & 0.5072 & 0.2071 & 103.5 & 0.4908 & 0.5216 & 3.325 & 3.559 & 1061.8 \\
\hline $2.20 \ldots \ldots \ldots \ldots$ & 1.371 & 3.989 & 0.358 & 0.2229 & 727.1 & 3.284 & 3.566 & 0.284 & 814.4 & 0.4401 & 0.103 & 0.4993 & 0.2002 & 113.8 & 0.4828 & 0.5153 & 3.341 & 3.561 & 943.5 \\
\hline $2.30 \ldots \ldots \ldots \ldots$ & 1.448 & 4.003 & 0.380 & 0.2366 & 637.8 & 3.133 & 3.583 & 0.285 & 706.5 & 0.4164 & 0.100 & 0.4869 & 0.2065 & 134.5 & 0.4685 & 0.5038 & 3.220 & 3.577 & 857.7 \\
\hline $2.45 \ldots \ldots \ldots \ldots$ & 1.554 & 4.023 & 0.422 & 0.2460 & 534.4 & 2.435 & 3.644 & 0.287 & 569.5 & 0.3293 & 0.035 & 0.4516 & 0.2064 & 264.4 & 0.4403 & 0.4820 & 3.126 & 3.589 & 859.3 \\
\hline $2.50 \ldots \ldots \ldots \ldots$ & 1.591 & 4.030 & 0.436 & 0.2543 & 502.3 & 2.466 & 3.643 & 0.287 & 531.7 & 0.3314 & 0.045 & 0.4620 & 0.2762 & 260.8 & 0.4443 & 0.4854 & 3.236 & 3.580 & 807.4 \\
\hline $2.60 \ldots \ldots \ldots \ldots$ & 1.657 & 4.042 & 0.473 & 0.2664 & 447.9 & 2.451 & 3.647 & 0.288 & 472.5 & 0.3373 & 0.060 & 0.4672 & 0.2435 & 228.5 & 0.4489 & 0.4888 & 3.115 & 3.594 & 720.1 \\
\hline $2.80 \ldots \ldots \ldots \ldots$ & 1.783 & 4.065 & 0.528 & 0.2900 & 360.9 & 2.458 & 3.651 & 0.289 & 378.1 & 0.3559 & 0.076 & 0.4863 & 0.2075 & 177.0 & 0.4701 & 0.5053 & 3.262 & 3.585 & 573.9 \\
\hline
\end{tabular}

a Initial mass (in solar units)

${ }^{b}$ Logarithm of the surface luminosity (in solar units) on the ZAMS

${ }^{c}$ Logarithm of the effective temperature (in K) on the ZAMS.

Mass of the convective core (in solar units) on the ZAMS

${ }^{\mathrm{e}} \mathrm{He}$ core mass (in solar units) at the end of the central H-burning phase

Central H-burning lifetime (in Myr).

Logarithm of the surface luminosity (in solar units) at the RGB tip.

${ }^{\mathrm{h}}$ Logarithm of the effective temperature (in K) at the RGB tip.

Surface He abundance (mass fraction) at the RGB tip.

Model age (in Myr) at the RGB tip.

${ }^{k} \mathrm{He}$ core mass (in solar units) at the beginning of the central He-burning phase.

Convective core mass (in solar mass units) at the beginning of the central He-burning phase.

${ }^{\mathrm{m}} \mathrm{He}$ core mass (in solar units) at the end of the central He-burning phase.

${ }^{\mathrm{n}} \mathrm{CO}$ core mass (in solar units) at the end of the central He-burning phase.

Central He-burning lifetime (in Myr).

$\mathrm{CO}$ core mass (in solar units) at the $1^{\circ}$ thermal pulse.

He core mass (in solar units) at the $1^{\circ}$ thermal pulse.

Logarithm of the surface luminosity (in solar units) at the $1^{\circ}$ thermal pulse.

Logarithm of the effective temperature (in K) at the $1^{\circ}$ thermal pulse.

${ }^{t}$ Model age (in Myr) at the beginning of the thermal pulses phase. 
TABLE 4

Selected Evolutionary and Structural Properties for Intermediate-Mass and Massive Models with [Fe/H] = 0.06 (No Overshooting)

\begin{tabular}{|c|c|c|c|c|c|c|c|c|c|c|c|c|c|c|c|c|c|}
\hline$M^{\mathrm{a}}$ & $\log L^{\mathrm{b}}$ & $\log T_{e}^{\mathrm{c}}$ & $M_{\mathrm{cc}}{ }^{\mathrm{d}}$ & $M_{\mathrm{He}}^{\mathrm{e}}$ & $t_{\mathrm{H}}^{\mathrm{f}}$ & $M_{\mathrm{He}}^{\mathrm{g}}$ & $M_{\mathrm{cc}}^{\mathrm{h}}$ & $M_{\mathrm{He}}{ }^{\mathrm{i}}$ & $M_{\mathrm{CO}}^{\mathrm{j}}$ & $t_{\mathrm{He}}{ }^{\mathrm{k}}$ & $M_{\mathrm{CO}}{ }^{1}$ & $\log L^{\mathrm{m}}$ & $M_{\mathrm{CO}}{ }^{\mathrm{n}}$ & $M_{\mathrm{He}}{ }^{\circ}$ & $\log L^{\mathrm{p}}$ & $\log T_{e}^{\mathrm{q}}$ & $\mathrm{Age}^{\mathrm{r}}$ \\
\hline $3.0 \ldots \ldots$. & 1.898 & 4.086 & 0.581 & 0.3123 & 296.6 & 0.3785 & 0.090 & 0.5134 & 0.2123 & 136.2 & No dredge-up & No dredge-up & 0.4989 & 0.5285 & 3.377 & 3.579 & 461.5 \\
\hline $3.5 \ldots \ldots$. & 2.151 & 4.132 & 0.749 & 0.3716 & 196.6 & 0.4393 & 0.109 & 0.6022 & 0.2386 & 75.6 & No dredge-up & No dredge-up & 0.5949 & 0.6124 & 3.717 & 3.557 & 287.2 \\
\hline $4.0 \ldots \ldots$. & 2.368 & 4.171 & 0.905 & 0.4322 & 138.1 & 0.5035 & 0.125 & 0.7169 & 0.2767 & 45.3 & No dredge-up & No dredge-up & 0.7131 & 0.7218 & 4.020 & 3.535 & 192.3 \\
\hline $4.5 \ldots \ldots$. & 2.558 & 4.204 & 1.068 & 0.4908 & 102.0 & 0.5712 & 0.139 & 0.8445 & 0.3882 & 29.7 & 0.7490 & 4.127 & 0.7794 & 0.7852 & 4.154 & 3.531 & 137.7 \\
\hline $5.0 \ldots \ldots$. & 2.724 & 4.232 & 1.242 & 0.5593 & 78.5 & 0.6403 & 0.171 & 0.9709 & 0.4303 & 21.4 & 0.7815 & 4.191 & 0.8036 & 0.8086 & 4.234 & 3.532 & 104.2 \\
\hline $5.5 \ldots \ldots \ldots$ & 2.872 & 4.258 & 1.402 & 0.6147 & 62.4 & 0.7121 & 0.199 & 1.1072 & 0.5070 & 16.6 & 0.8181 & 4.242 & 0.8311 & 0.8354 & 4.276 & 3.535 & 82.0 \\
\hline $6.0 \ldots \ldots$. & 3.007 & 4.280 & 1.425 & 0.6838 & 51.0 & 0.7886 & 0.221 & 1.2314 & 0.5273 & 12.7 & 0.8433 & 4.269 & 0.8539 & 0.8576 & 4.302 & 3.540 & 65.6 \\
\hline $6.5 \ldots \ldots$. & 3.127 & 4.301 & 1.601 & 0.7472 & 42.6 & 0.8666 & 0.255 & 1.3688 & 0.6035 & 10.3 & 0.8799 & 4.315 & 0.8887 & 0.8915 & 4.375 & 3.538 & 54.4 \\
\hline $7.0 \ldots \ldots$. & 3.238 & 4.320 & 1.783 & 0.8124 & 36.3 & 0.9475 & 0.277 & 1.5144 & 0.5827 & 8.7 & 0.9249 & 4.337 & 0.9366 & 0.9386 & 4.529 & 3.530 & 46.1 \\
\hline $7.5 \ldots \ldots$. & 3.339 & 4.337 & 1.978 & 0.9084 & 31.5 & 1.0401 & 0.294 & 1.6383 & 0.6996 & 7.0 & 0.9475 & 4.362 & 0.9589 & 0.9605 & 4.643 & 3.522 & 39.4 \\
\hline $8.0 \ldots \ldots$. & 3.433 & 4.352 & 2.168 & 0.9878 & 27.7 & 1.1337 & 0.327 & 1.7828 & 0.7936 & 5.9 & 0.9806 & 4.275 & Carbon ignition & Carbon ignition & Carbon ignition & Carbon ignition & Carbon ignition \\
\hline $8.5 \ldots \ldots$. & 3.520 & 4.367 & 2.376 & 1.0969 & 24.6 & 1.2343 & 0.373 & 1.9304 & 0.8474 & 5.0 & 1.0310 & 4.1886 & Carbon ignition & Carbon ignition & Carbon ignition & Carbon ignition & Carbon ignition \\
\hline $9.0 \ldots \ldots$. & 3.601 & 4.380 & 2.593 & 1.1884 & 22.1 & 1.3390 & 0.402 & 2.0970 & 1.0264 & 4.5 & No dredge-up & No dredge-up & Carbon ignition & Carbon ignition & Carbon ignition & Carbon ignition & Carbon ignition \\
\hline $9.5 \ldots \ldots$. & 3.677 & 4.393 & 2.806 & 1.3012 & 20.0 & 1.4588 & 0.454 & 2.2517 & 1.1457 & 4.2 & No dredge-up & No dredge-up & Carbon ignition & Carbon ignition & Carbon ignition & Carbon ignition & Carbon ignition \\
\hline $10.0 \ldots \ldots$ & 3.749 & 4.404 & 3.038 & 1.4394 & 18.3 & 1.5752 & 0.491 & 2.3731 & 1.1396 & 3.7 & No dredge-up & No dredge-up & Carbon ignition & Carbon ignition & Carbon ignition & Carbon ignition & Carbon ignition \\
\hline
\end{tabular}

${ }^{a}$ Initial mass (in solar units).

${ }^{b}$ Logarithm of the surface luminosity (in solar units) on the ZAMS

${ }^{c}$ Logarithm of the effective temperature (in K) on the ZAMS.

d Mass of the convective core (in solar units) on the ZAMS.

${ }^{\mathrm{e}} \mathrm{He}$ core mass (in solar units) at the end of the central H-burning phase.

Central H-burning lifetime (in Myr).

He core mass (in solar units) at the beginning of the central He-burning phase.

Mass of the convective core (in solar units) at the beginning of the central He-burning phase.

He core mass (in solar units) at the end of the central He-burning phase.

$\mathrm{CO}$ core mass (in solar units) at the end of the central He-burning phase.

${ }^{k}$ Central He-burning lifetime (in Myr).

$\mathrm{CO}$ core mass (in solar units) at the $2^{\circ}$ dredge-up.

${ }^{\mathrm{m}}$ Logarithm of the surface luminosity (in solar units) at the $2^{\circ}$ dredge-up.

${ }^{\mathrm{n}} \mathrm{CO}$ core mass (in solar units) at the $1^{\circ}$ thermal pulse.

He core mass (in solar units) at the $1^{\circ}$ thermal pulse.

${ }^{\mathrm{p}}$ Logarithm of the surface luminosity (in solar units) at the $1^{\circ}$ thermal pulse.

${ }^{\mathrm{q}}$ Logarithm of the effective temperature (in $\mathrm{K}$ ) at the $1^{\circ}$ thermal pulse.

Model age (in Myr) at the beginning of the thermal pulses phase. 


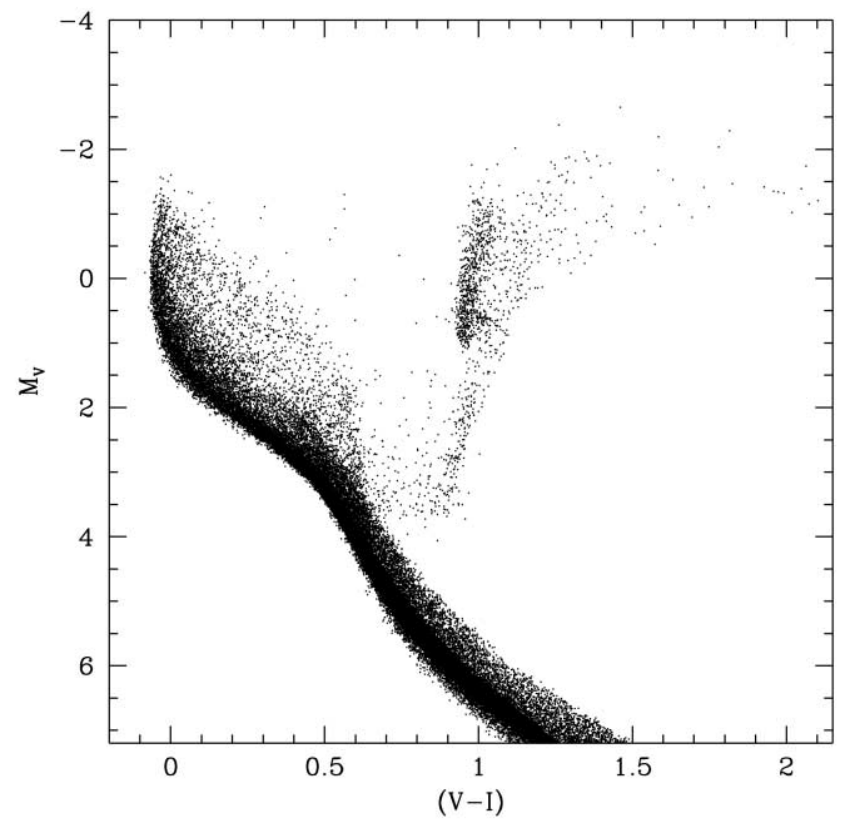

FIG. 6.-Example of CMD produced by our code SYNTHETIC MAN for a composite stellar population (see text for details).

described in Castelli (1998, 1999), and Bessell et al. (1998). Updated grids of ATLAS9 model atmospheres and fluxes (Castelli \& Kurucz 2003) were used to generate synthetic colors and bolometric corrections needed to transform from the theoretical to the observational planes.

\subsubsection{The Model Atmospheres}

New grids of ATLAS9 model atmospheres were computed for solar and several scaled solar metallicities. For this paper we used grids with metallicities $[M / \mathrm{H}]$ equal to $+0.5,+0.2$, $0.0,-0.5,-1.0,-1.5,-2.0$, and $-2.5 .^{11}$ For all the models the microturbulent velocity is $\xi=2 \mathrm{~km} \mathrm{~s}^{-1}$. The new grids, called ODFNEW, differ from the previous ones quoted as K95 and C97 in Castelli (1999) as follows:

1. The number of models is 476 in all the grids. They range from 3500 to $50,000 \mathrm{~K}$ in $T_{\text {eff }}$ and from 0.0 to 5.0 in $\log g$.

2. All the models have the same number of 72 planeparallel layers from $\log \tau_{\text {Ross }}=-6.875$ to +2.00 at steps of $\Delta \log \tau_{\text {Ross }}=0.125$.

3. All the models are computed with the updated solar abundances from Grevesse \& Sauval (1998) $)^{12}$ and therefore with updated opacity distribution functions, which now also include $\mathrm{H}_{2} \mathrm{O}$ lines.

4. All the models are computed with the convection option switched on and with the overshooting option switched off. Mixing-length convection with $l / H_{P}=1.25$ is assumed for all the models. The convective flux decreases with increasing $T_{\text {eff }}$, and it naturally becomes negligible for $T_{\text {eff }}$ on the order of $9000 \mathrm{~K}$.

5. The quasi-molecular absorption $\mathrm{H}-\mathrm{H}^{+}$was added to the opacity. It affects the ultraviolet flux of metal-poor A-type stars (Castelli \& Cacciari 2001).

\footnotetext{
${ }^{11}[\mathrm{M} / \mathrm{H}]$ denotes the difference in $\log (\mathrm{Z} / H)$ with respect to the solar value; for a scaled solar metal distribution $[\mathrm{M} / \mathrm{H}]=[\mathrm{Fe} / \mathrm{H}]$.

${ }_{12}$ This metal distribution is slightly different from the one adopted for the stellar evolution computation; however, the difference between the two distributions is small and does not cause any appreciable inconsistency.
}

The largest differences between these models and the previous ones occur for $T_{\text {eff }} \leq 4500 \mathrm{~K}$, mostly because of the addition of the $\mathrm{H}_{2} \mathrm{O}$ contribution to the line opacity.

\subsubsection{The Colors}

The $U$ passband is from Buser (1978), while the $B$ and $V$ passbands are from Azusienis \& Straižys (1969). The $R$ and $I$ Cousins colors have passbands from Bessell (1990). The JKL colors were computed with passbands from Johnson (1965) reported also by Lamla (1982). For the $H$ color the passband from Bessell \& Brett (1988) was adopted.

Zero points for the $V$ magnitude and for $(U-B),(B-V)$, $(V-I),(V-R),(V-J),(V-K)$, and $(V-L)$ color indices were set by normalizing the computed colors for Vega to the observed colors. The Vega model atmosphere is from Castelli $\& \operatorname{Kurucz}\left(1994 ; T_{\text {eff }}=9550 \mathrm{~K}, \log g=3.95,[M / \mathrm{H}]=-0.5\right.$, $\left.\xi=2.0 \mathrm{~km} \mathrm{~s}^{-1}\right)$; the Vega observed $V$ magnitude and colors are $V=0.03,(U-B)=0.0$, and $(B-V)=0.0$ (Johnson et al. 1966), $(V-I)=-0.005$ and $(V-R)=-0.009$ (Bessell 1983), $(V-J)=0.0,(V-K)=0.0$, and $(V-L)=$ 0.0 . For $(H-K)$ the computed index of Sirius was normalized to the observed one $(H-K)=-0.009$ (I. Glass 1997, private communication). The Sirius model is from R. L. Kurucz (1997, private communication ${ }^{13} T_{\mathrm{eff}}=9850 \mathrm{~K}, \log g=4.30$, $\left.[\mathrm{M} / \mathrm{H}]=+0.4, \xi=0.0 \mathrm{~km} \mathrm{~s}^{-1}, \mathrm{He} / \mathrm{H}=+0.5\right)$.

After some preliminary comparisons with open cluster MS loci, we have decided to use the empirical color- $T_{\text {eff }}$ relationships provided by Houdashelt et al. (2000) only for MS stars with $T_{\text {eff }} \leq 4750 \mathrm{~K}$, and only for $(B-V),(V-I)$, and $(V-R)$ colors. The bolometric correction $\mathrm{BC}_{V}$ is given by

$$
\mathrm{BC}_{V}=-2.5\left\{\log \left(\sigma T_{\text {eff }}^{4} / \pi\right)-\log \left[\int_{\alpha}^{\beta} S_{V}(\lambda) F(\lambda) d \lambda\right]\right\}+K,
$$

where $S_{V}(\lambda)$ is the $V$ passband and $F(\lambda)$ is the computed flux. The constant $K$ was defined by normalizing to zero the smallest bolometric correction (in absolute value) of the whole synthetic grid computed for $[\mathrm{M} / \mathrm{H}]=0.0$ and microturbulent velocity $\xi=2.0 \mathrm{~km} \mathrm{~s}^{-1}$. It occurs for the model with $T_{\text {eff }}=7250 \mathrm{~K}, \log g=0.5$. With this normalization, positive values for $\mathrm{BC}_{V}$ are avoided. The value of $\mathrm{BC}_{V \odot}$ is -0.203 for a solar model with parameters $T_{\text {eff }}=5777 \mathrm{~K}, \log g=4.4377$, and $\xi=1.0 \mathrm{~km} \mathrm{~s}^{-1}$.

By assuming $V_{\odot}=-26.75$ (Hayes 1985), the solar absolute magnitude is $M_{V \odot}=4.82$. Therefore, for the adopted normalization, $M_{\text {bol } \odot}$ is given by $M_{V \odot}+\mathrm{BC}_{V \odot}=4.62$.

All the ODFNEW grids of models and colors are available on the Web. ${ }^{14}$ In Figure 7 we display a comparison of our $500 \mathrm{Myr}$ (including overshooting) and $10 \mathrm{Gyr}[\mathrm{Fe} / \mathrm{H}]=0.06$ isochrones, transformed to the $M_{V}-(V-I)$ CMD by using our adopted transformations discussed before, plus the Yale (Green 1988), NEXTGEN (Allard et al. 1997), and older ATLAS 9 (Castelli 1999) transformations. This comparison is representative of the general differences in the various color planes covered by our isochrones. The Yale transformations tend to produce a bluer, lower MS, RGB, and He-burning phase, whereas the cooler and brighter part of the RGB tends to agree with our results. Color differences can be as high as $\sim 0.2$ mag. The NEXTGEN transformations are available for

\footnotetext{
${ }^{13}$ See http://kurucz.harvard.edu/stars/SIRIUS.

14 See http://kurucz.harvard.edu/grids.html and http://www.user.oat.ts.astro .it/castelli/grids.html.
} 


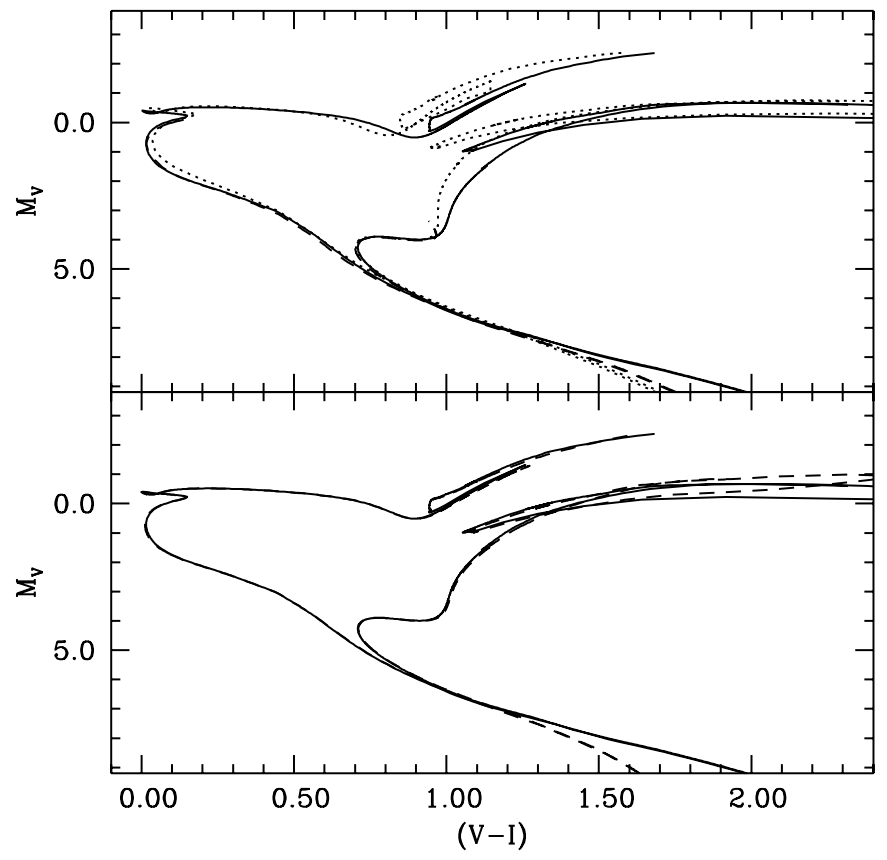

FIG. 7.-Comparison between our [Fe/H] 0.06 isochrones of $500 \mathrm{Myr}$ (including overshooting) and $10 \mathrm{Gyr}$ transformed to the $M_{V}-(V-I)$ CMD by using various sets of color transformations, i.e., the transformations adopted in this paper (solid line), the Yale transformations by Green (1988, upper panel, dotted line), NEXTGEN transformations by Allard et al. (1997, upper panel, dashed line), and the earlier ATLAS 9 transformations by Castelli (1999, lower panel, dashed line).

surface gravities higher than or equal to $\log g=3.5$ (in $\operatorname{cgs}$ units) and differ (bluer) only on the lower MS. The earlier ATLAS 9 transformations are very similar apart from the lower MS and cooler RGB, where color differences of $\sim 0.2$ mag or more are reached.

\section{COMPARISON WITH EXISTING DATABASES}

In this section we compare the H-R diagram of selected isochrones with the counterparts from the publicly available databases by Girardi et al. (2000), Castellani et al. (2003), Lejeune \& Schaerer (2001), and Yi et al. (2001). In addition, we add a comparison with Bergsbusch \& Vandenberg (2001) isochrones kindly provided by the authors (D. A. VandenBerg 2003, private communication).

These various grids of models are computed employing some different choices of the physical inputs (opacities are usually the same, but EOS, nuclear reaction rates, boundary conditions, and neutrino energy loss rates are often different), and also the chemical compositions are generally different. We perform the comparison on the theoretical H-R diagram, thus bypassing the additional degree of freedom introduced by the choice of the color transformations. We employed our isochrones including overshooting (unless otherwise stated), since all these sets of isochrones are computed including overshooting from the convective cores, albeit often with different prescriptions about its extension and the decrease to zero for decreasing stellar mass.

We have selected isochrone ages that span all the relevant age range and chemical compositions as close as possible to our choices. It is important to note that Castellani et al. (2003) and Yi et al. (2001) models do include atomic diffusion (He and metals for Castellani et al. models, only He for Yi et al.

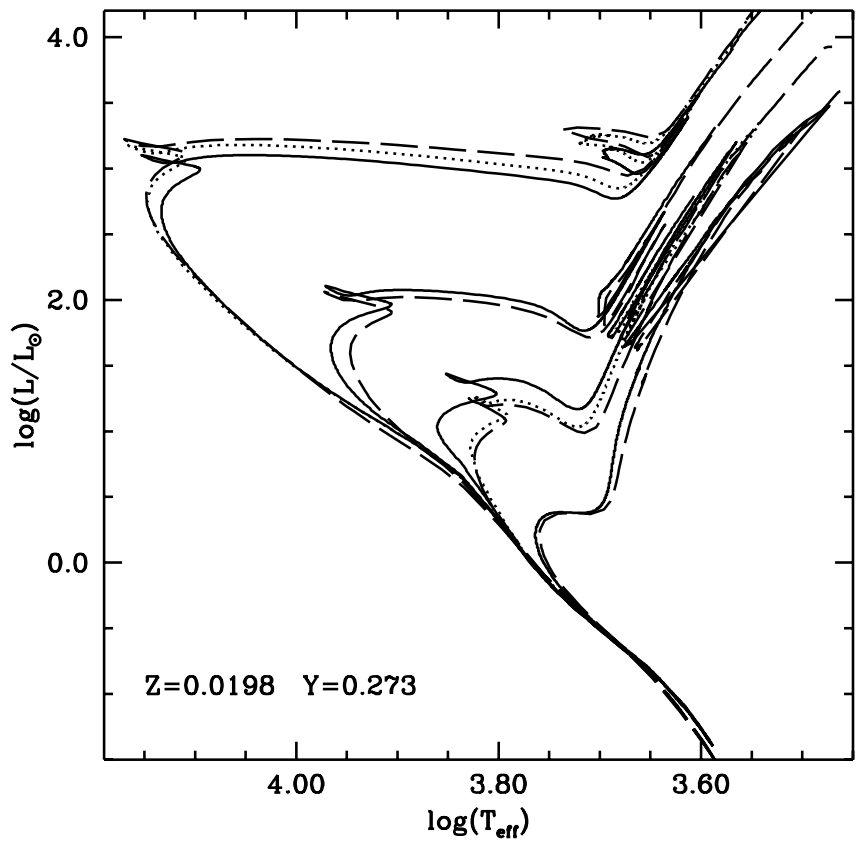

FIG. 8.-Comparison between our isochrones (solid lines) and Girardi et al. (2000) isochrones (dashed lines) for selected ages (100 Myr, $500 \mathrm{Myr}, 1.8 \mathrm{Gyr}$, and $10 \mathrm{Gyr}$ ). The chemical composition of our isochrones is as labeled; Girardi et al. (2000) isochrones have $Y=0.273$ and $Z=0.019$. Dotted lines show our isochrones for ages of $90 \mathrm{Myr}$ and $2.5 \mathrm{Gyr}$, respectively.

models), and therefore their isochrones for old ages (above a few Gyr) are affected by the efficiency of this process.

Figure 8 shows a comparison with the Girardi et al. (2000) isochrones; the chemical composition of our isochrones is as labeled, very close to the $Z=0.019, Y=0.273$ composition of Girardi et al. (2000) isochrones. The selected ages are $100 \mathrm{Myr}, 500 \mathrm{Myr}$, $1.8 \mathrm{Gyr}$, and $10 \mathrm{Gyr}$, respectively. The MS loci do agree reasonably well, whereas our RGBs are systematically hotter, apart from the youngest isochrone. Our He-burning models are slightly brighter, especially for the $500 \mathrm{Myr}$ isochrone, but become fainter at $100 \mathrm{Myr}$. The TO regions are in good agreement at $10 \mathrm{Gyr}$; our TO models become brighter at $1.8 \mathrm{Gyr}$ and have the same luminosity as Girardi et al. (2000), but they are hotter at $500 \mathrm{Myr}$ and fainter at 100 Myr. At 1.8 Gyr the TO region of Girardi et al. (2000) isochrones is reproduced if we select an age higher by $0.7 \mathrm{Gyr}$ (e.g., the mass evolving at the TO of our isochrone is higher than the Girardi et al. 2000 counterpart); at 100 Myr we need to choose an age $10 \mathrm{Myr}$ younger to reproduce the TO of Girardi et al. (2000) isochrones. Since Girardi et al. (2000) also computed a set of canonical isochrones for this metallicity, we have investigated in more detail the reason for the relatively large difference at 1.8 Gyr. We discovered that the difference between the 1.8 Gyr old canonical isochrones - which are only due to the difference in the model input physics - accounts for about $40 \%$ of the discrepancy between the overshooting models. The remaining fraction of the discrepancy has to be ascribed to the interplay between the overshooting treatment and the different input physics.

In Figure 9 we display a comparison with selected isochrones without overshooting and initial $Z=0.008$ and $Y=0.250$, from the database by Castellani et al. (2003). The agreement with our canonical models is quite good all along the MS, TO region, and RGB. Our He-burning luminosities are slightly lower, and at $10 \mathrm{Gyr}$ there is a larger difference at 


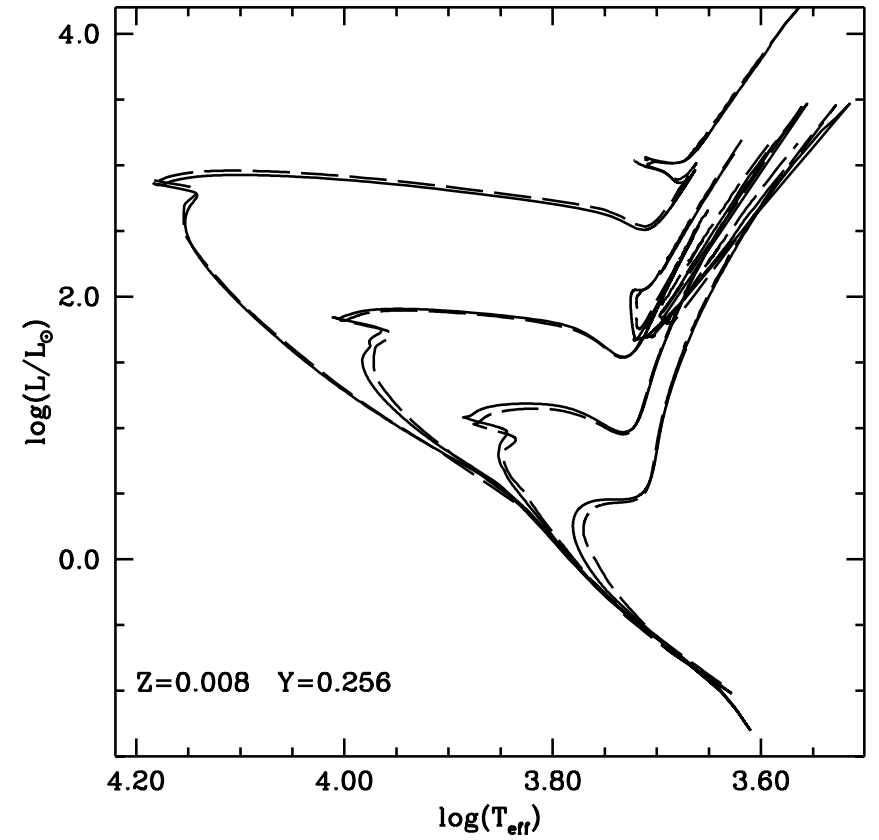

FIG. 9.- Same as Fig. 8, but for the isochrones by Castellani et al. (2003) with $Y=0.250$ and $Z=0.008$.

the TO region due to the effect of atomic diffusion included by Castellani et al. (2003). We do not find a substantial discrepancy in the TO brightness at $1.8 \mathrm{Gyr}$, as in the case of the comparison with the canonical models by Girardi et al. (2000).

The comparison with Lejeune \& Schaerer (2001) models with initial $Z=0.004$ and $Y=0.252$ is displayed in Figure 10 . Ages are the same as in the previous comparison. The isochrone MSs of the two sets look in reasonable agreement, and the TO region of the oldest and youngest isochrones are practically identical. However, for the $500 \mathrm{Myr}$ and $1.8 \mathrm{Gyr}$ the TO of our isochrones is clearly brighter. At 1.8 Gyr we should

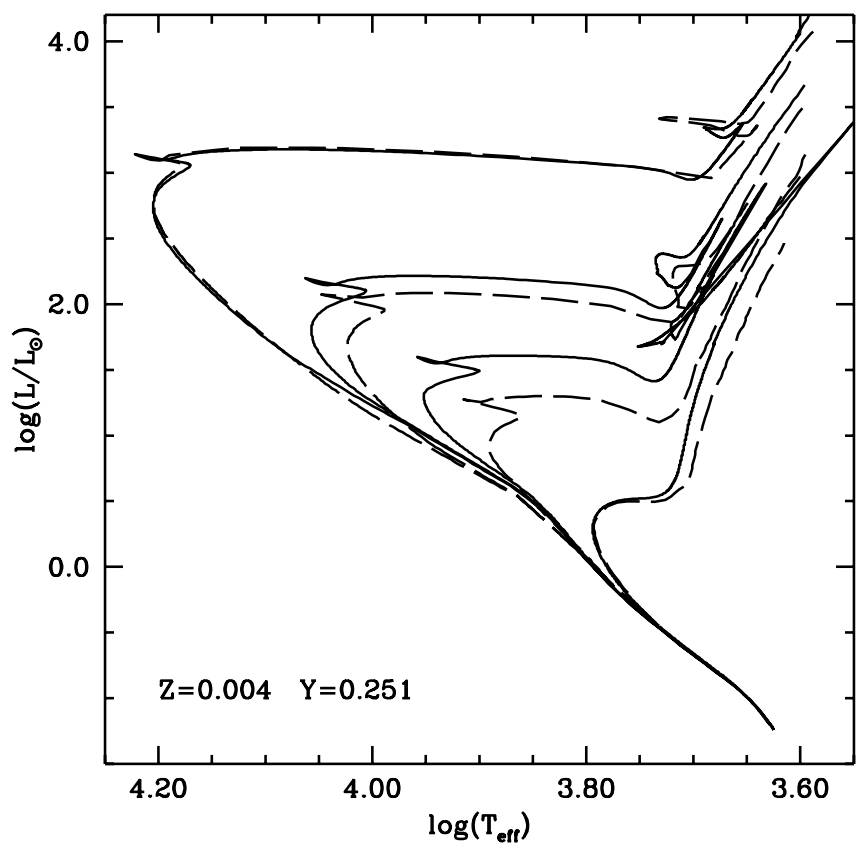

FIG. 10.-Same as Fig. 8, but for the isochrones by Lejeune \& Schaerer (2001) with $Y=0.252$ and $Z=0.004$.

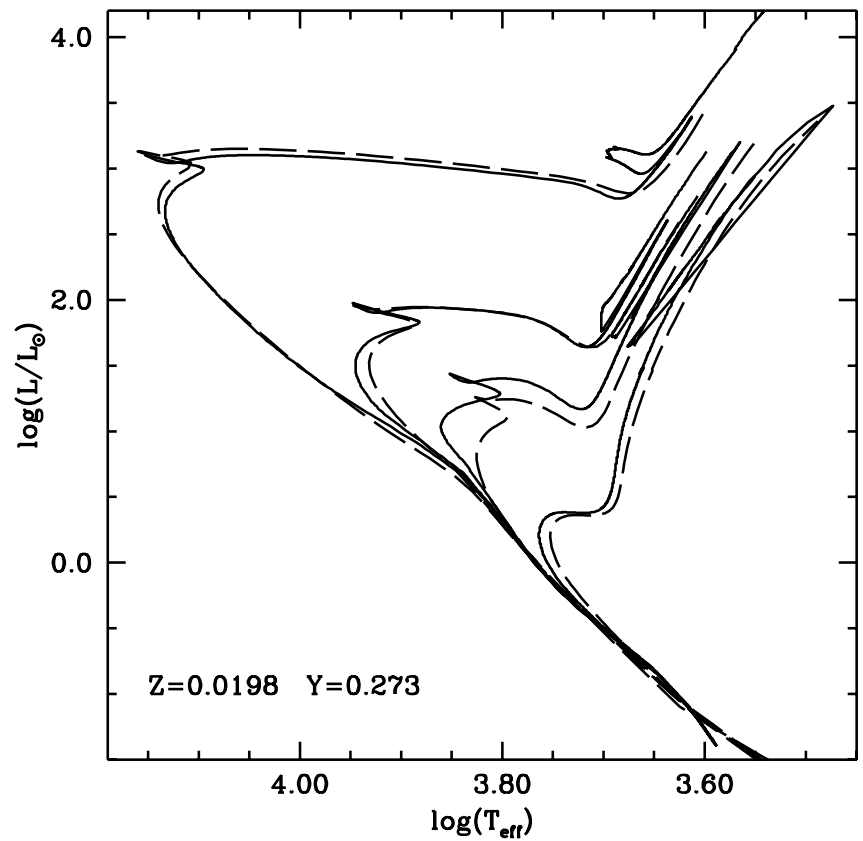

FIG. 11.- Same as Fig. 8, but for the isochrones by Yi et al. (2001) with $Y=0.27$ and $Z=0.02$. Selected ages are $100 \mathrm{Myr}, 600 \mathrm{Myr}, 1.8 \mathrm{Gyr}$, and 10 Gyr, respectively.

employ an isochrone about $1 \mathrm{Gyr}$ older in order to match the Lejeune \& Schaerer (2001) isochrone. Our RGBs are much hotter, and the He-burning phase (when available in Lejeune \& Schaerer 2001 isochrones) is brighter at $500 \mathrm{Myr}$ and fainter at 100 Myr.

In the case of the Yi et al. (2001) database, we compared with the $Z=0.02, Y=0.27$ isochrones for ages of $100 \mathrm{Myr}$, $600 \mathrm{Myr}, 1.8 \mathrm{Gyr}$, and $10 \mathrm{Gyr}$ (see Fig. 11). Also, in this case the MS loci appear to be in good agreement, apart from the lower luminosities, where the Yi et al. (2001) models show a different slope. The TO of their $10 \mathrm{Gyr}$ isochrone is affected by diffusion and behaves accordingly when compared with our models. At 100 and 500 Myr the brightness of the TO regions is similar, although effective temperatures are slightly different; at $1.8 \mathrm{Gyr}$ there is again a difference in the TO luminosity that translates into an age difference of about $800 \mathrm{Myr}$, as in the case of the comparison with Girardi et al. (2000) isochrones; our RGBs are hotter at all ages.

The comparison with VandenBerg isochrones $(Y=0.277$ and $Z=0.0188$ ) is shown in Figure 12 for ages of 2 and 10 Gyr. The 10 Gyr isochrones do agree very well also along the RGB. At 2 Gyr we find the same differences encountered in the other comparisons.

A common result of the previous discussion is the fact that our RGB temperatures are generally hotter than the others, and the TO luminosity for our $1.8 \mathrm{Gyr}$ isochrones is higher. As far as the RGB temperatures are concerned, the difference is certainly not due (apart from the comparison with Vandenberg models, where the RGB location is exactly the same in the 10 Gyr isochrones that have the same RGB evolving mass, and with Castellani et al. models) to different values of the mass evolving along the RGB (higher masses when our isochrones are brighter at a given age), since we find this difference also with respect to isochrones with the same RGB evolving mass. We see in $\S 7$ that this difference in the RGB temperatures (possibly due to a combination of different EOS and solar mixing-length calibration obtained using different boundary 


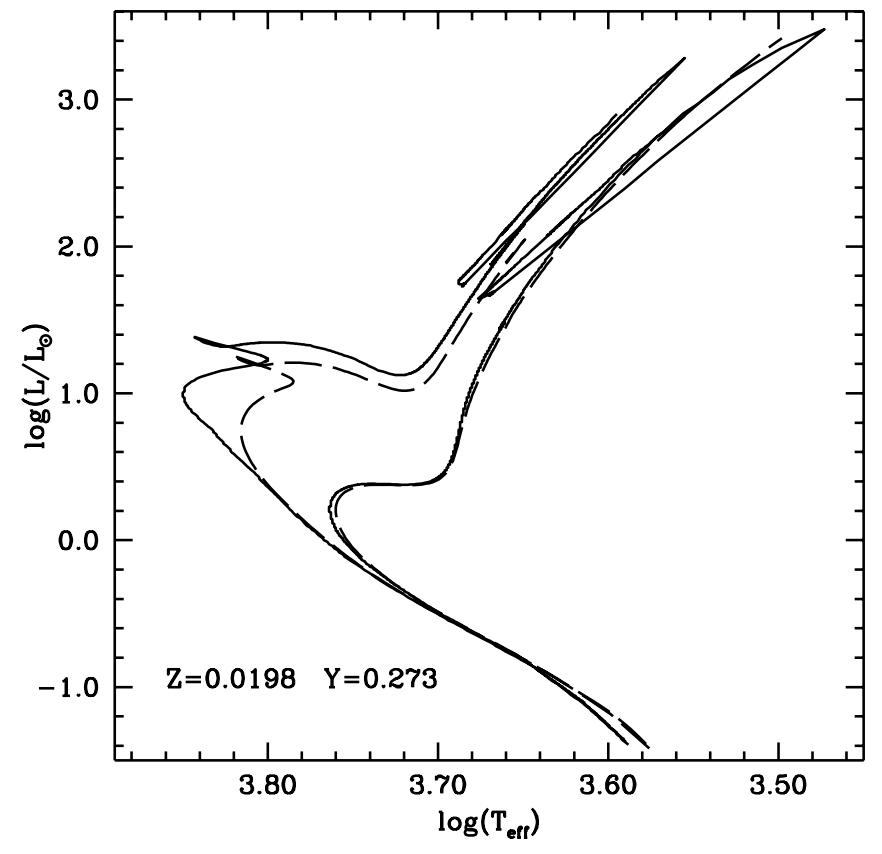

FIG. 12.-Same as Fig. 8, but for D. A. VandenBerg (2003, private communication) isochrones with $Y=0.277$ and $Z=0.0188$. Selected ages are 2 and $10 \mathrm{Gyr}$, respectively.

conditions; see, e.g., Salaris et al. 2002)does not prevent our models from fitting very well the observed RGBs in a sample of Galactic open clusters when coupled to our color transformations. To better justify why we think these effective temperature discrepancies along the RGB may be due to a combination of different boundary conditions and EOS, we note that Salaris et al. (2002) have shown how different boundary conditions [e.g., the gray vs. Krishna Swamy $T(\tau)$ relationship] produce different solar mixing-length calibrations that make all solar models agree at the Sun location but disagree along the RGB by differences on the order of $100 \mathrm{~K}$. As for the influence of the EOS, the same $1 M_{\odot}$ solar composition models discussed in $\S 2$, computed using the Irwin EOS and the EOS employed in Cassisi \& Salaris (1997) keeping everything else unchanged, show $T_{\text {eff }}$ differences along the RGB on the order of $\sim 280 \mathrm{~K}$ at the base of the RGB, $\sim 140 \mathrm{~K}$ at $\log \left(L / L_{\odot}\right)=2$, and $\sim 85 \mathrm{~K}$ at the He flash ignition, in the sense of having hotter temperatures with the Irwin EOS.

As for the TO brightness differences at 1.8 Gyr (which, however, is negligible in the case of the comparison with Castellani et al. 2003 models without overshooting), it persists for ages higher than $1.8 \mathrm{Gyr}$, up to when the overshooting distance and the size of the convective cores are reduced to zero (ages of about 5-6 Gyr). We believe that this might be related mainly to the different MS lifetime caused by the use of a different EOS, coupled to differences in the way the overshooting distance is reduced to zero when approaching the radiative core regime.

To conclude this section, we show in Figure 13 a comparison between our ZAHB luminosity at the instability strip versus $[\mathrm{Fe} / \mathrm{H}]$ theoretical relationship and analogous results from various authors. Our new values are systematically brighter than Cassisi \& Salaris (1997) models, mainly as a result of the effect of the new plasma neutrino rates and the higher initial He content (in Cassisi \& Salaris 1997 we employed a primordial He mass fraction equal to 0.23 ). The main difference in the Cassisi et al. (1998) models is the EOS for the electron

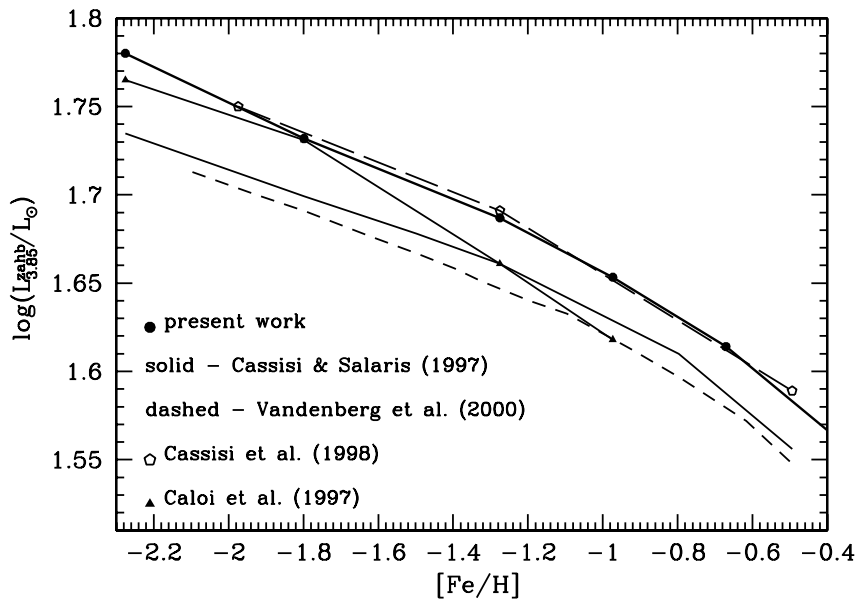

FIG. 13.-Comparison of the labeled theoretical relationships between the ZAHB luminosity at the instability strip $\left(\log T_{\text {eff }}=3.85\right)$ and $[\mathrm{Fe} / \mathrm{H}]$.

degenerate cores along the RGB, which increases the He flash core mass with respect to our result (they also used the I93 electron conduction opacities that slightly increase the He core mass in this metallicity range), but this increase is offset by the fact that their adopted He abundance is lower. Vandenberg et al. (2000) models are the faintest ones of this group, and we believe this is mainly due to their use of the Hubbard \& Lampe (1969) electron conduction opacities (which produce a lower He core mass at the He flash with respect to our adopted opacities), coupled to a lower primordial He abundance. The results by Caloi et al. (1997) have an overall different slope with respect to the other models, and we are not able to exactly pinpoint the reason for this difference.

\section{EMPIRICAL TESTS}

In this section we present the results of some empirical test we performed in order to establish the consistency of our models with photometric constraints. We have successfully tested our models in Cassisi et al. (2003) and Riello et al. (2003), in the regime of Galactic globular clusters. In particular, we have shown that with our models the initial He abundance of globular clusters as estimated from the $R$-parameter is finally in agreement with the cosmological constraint (Cassisi et al. 2003). More tests on Galactic globular cluster stars (whose initial chemical composition is characterized by $[\alpha / \mathrm{Fe}]>0$ ) will be presented in the companion paper with the $\alpha$-enhanced model grid. The reason for this is twofold. First, scaled solar isochrones are not an appropriate surrogate for $\alpha$-enhanced ones with the same $Z$ when $Z$ is above $\approx 0.002$ (e.g., Salaris $\&$ Weiss 1998); second, from our new $\alpha$-enhanced color transformations we have found that, for example, scaled solar $(B-V)$-effective temperature relationships differ from $\alpha$-enhanced ones, starting from relatively low metallicities around $Z \sim 0.001$.

In the comparisons detailed below, we employed the CMDs of field stars and a sample of clusters with empirically established parameters such as distance, $[\mathrm{Fe} / \mathrm{H}]$ (and scaled solar metal mixture), and reddening. In this way we do not have much freedom to change the parameters of our models in order to match the observations. Comparisons with, for example, Large Magellanic Cloud (LMC) clusters would be much less meaningful, since reddenings, $[\mathrm{Fe} / \mathrm{H}]$ (especially), and distances (not only the distance to the LMC barycenter, but also geometrical effects) are not well established empirically. We 


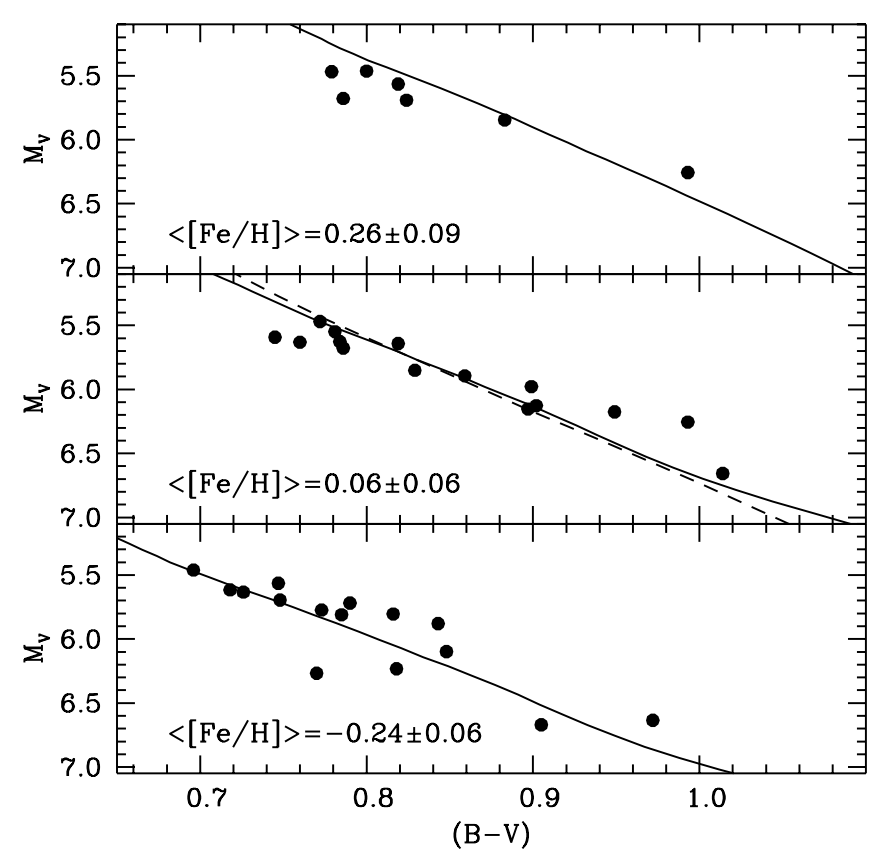

FIg. 14.-Comparison in the $M_{V}-(B-V)$ CMD between the unevolved MS of our isochrones and field dwarfs with the labeled mean metallicities (see text for details). The dashed line refers to the Girardi et al. (2000) isochrone with $[\mathrm{Fe} / \mathrm{H}]=0.06$.

could fit the CMDs of those clusters and give our estimates of their distance and reddening by playing with combinations of reddening, distance, and $[\mathrm{Fe} / \mathrm{H}]$, but this would not be a real test for the accuracy of the models.

We also refrained from comparing theoretical integrated colors with galactic open clusters or LMC data. The problem is that integrated colors of galactic open clusters are very uncertain as a result of their low total mass that induces large stochastic color fluctuations preventing any meaningful comparisons (see, e.g., the numerical experiments by Bruzual \& Charlot 2003 and the integrated colors reported in the WEBDA database (see Mermilliod 1992). ${ }^{15}$ The same applies to LMC clusters, with the additional problems of uncertain age and reddening. The situation is better for the more massive Galactic globular clusters, and in fact we will perform (using the appropriate $\alpha$-enhanced theoretical isochrones and color transformations) this comparison in our forthcoming paper about $\alpha$-enhanced models. In the following comparisons, when necessary, we use the reddening law $A_{V}=3.24 E(B-V), A_{I}=$ $1.96 E(B-V)$, and $A_{K}=0.11 A_{V}$ (Schlegel et al. 1998).

\subsection{Solar Neighborhood MS Stars}

Percival et al. (2002) have published homogeneous multicolor photometry and photometric metallicity estimates for a large sample of local field dwarfs with accurate Hipparcos parallaxes; the $[\mathrm{Fe} / \mathrm{H}]$ values of the individual stars range from $\sim-0.4$ up to +0.3 . These data are extraordinarily well suited to test the MS location of our theoretical isochrones in various color bands; moreover, the choice of the isochrone age is irrelevant, since the observed stars are all unevolved, i.e., fainter than $M_{V} \sim 5.5$.

We have divided the Percival et al. (2002) sample into three subsamples, as follows. The most metal-poor subsample comprises objects with $[\mathrm{Fe} / \mathrm{H}]$ between -0.35 and -0.15 , with

\footnotetext{
${ }^{15}$ See http://obswww.unige.ch/webda/integre.html.
}

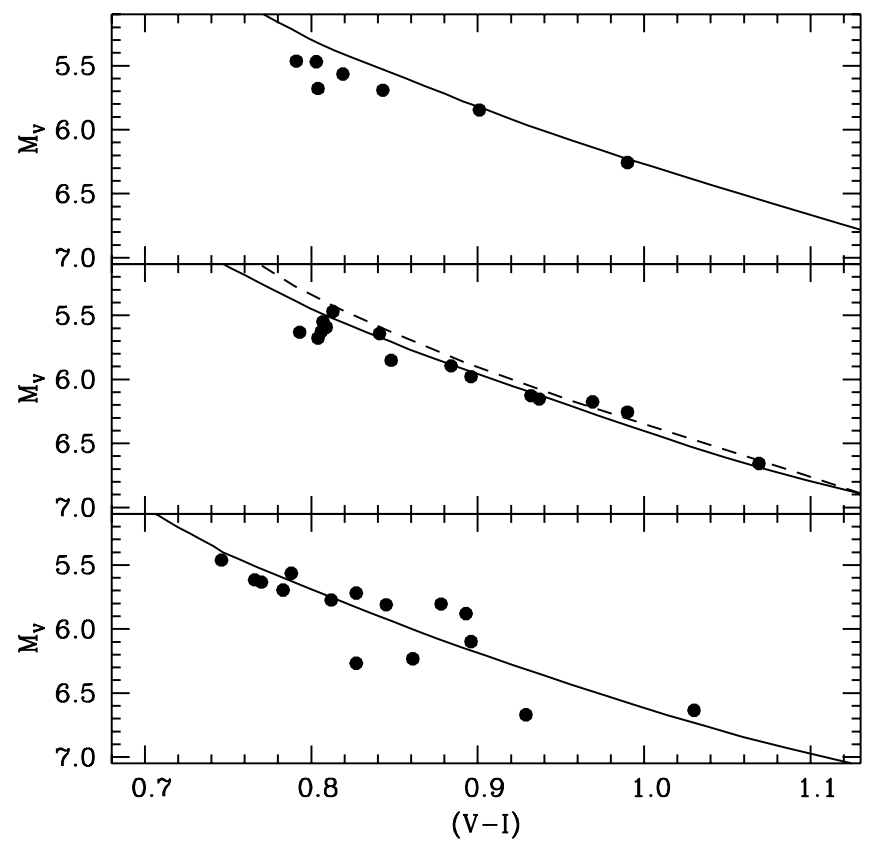

FIG. 15.-Same as Fig. 14, but for the $M_{V}-(V-I)$ CMD.

an average value of $[\mathrm{Fe} / \mathrm{H}]=-0.24 \pm 0.06$; the intermediate subsample contains stars with $[\mathrm{Fe} / \mathrm{H}]$ between -0.05 and +0.15 , with an average value of $0.06 \pm 0.06$. The third subsample is made of the most metal-rich objects, with $[\mathrm{Fe} / \mathrm{H}]$ between 0.16 and 0.36 , and an average $[\mathrm{Fe} / \mathrm{H}]$ equal to $0.26 \pm 0.09$. In Figures 14-18 we compare the CMD of these three subgroups with our isochrones for $[\mathrm{Fe} / \mathrm{H}]=-0.25$, 0.06 , and +0.26 , respectively. The comparison is made in the $M_{V}-(B-V), M_{V}-(V-I), M_{V}-(V-R)$ and $M_{K}-(V-$ $K), M_{K}-(J-K)$ planes; the $J$ and $K$ magnitudes of the field stars come from the 2MASS database (M. Groenewegen \& S. Percival 2003, private communication) and have been transformed to our adopted IR system following Carpenter (2001). For the sake of comparison we also show a Girardi et al. (2000) isochrone with $[\mathrm{Fe} / \mathrm{H}]=0.06$.

The agreement between the empirical CMDs and the isochrones appears to be satisfactoryin the sense that the theoretical isochrones usually overlap the main CMD locus occupied by the dwarfs in the various metallicity bins and color planes, although it tends to deteriorate slightly - apart from the case of $M_{K}-(V-K)$-at the highest metallicity (where, however, the star sample is the smallest).

\subsection{Open Clusters}

We have employed a sample of galactic open clusters of various ages for further tests of the reliability of the magnitudes and colors of our isochrones. In more detail, we have considered the CMDs of the Hyades (Pinsonneault et al. 2003), NGC 2420 (Anthony-Twarog et al. 1990), M67 (Sandquist 2004), and NGC 188 ( $B V$ from Caputo et al. 1990 and VI from von Hippel \& Sarajedini 1998), for which precise parallaxbased distance determinations do exist. In the case of the Hyades, accurate parallaxes of the individual stars in the observed CMD have been determined by the Hipparcos satellite. For the other clusters the distances have been determined by Percival \& Salaris (2003, hereafter PS03) using a completely empirical MS-fitting method presented in Percival et al. (2002). The MS template sequences used in this technique 


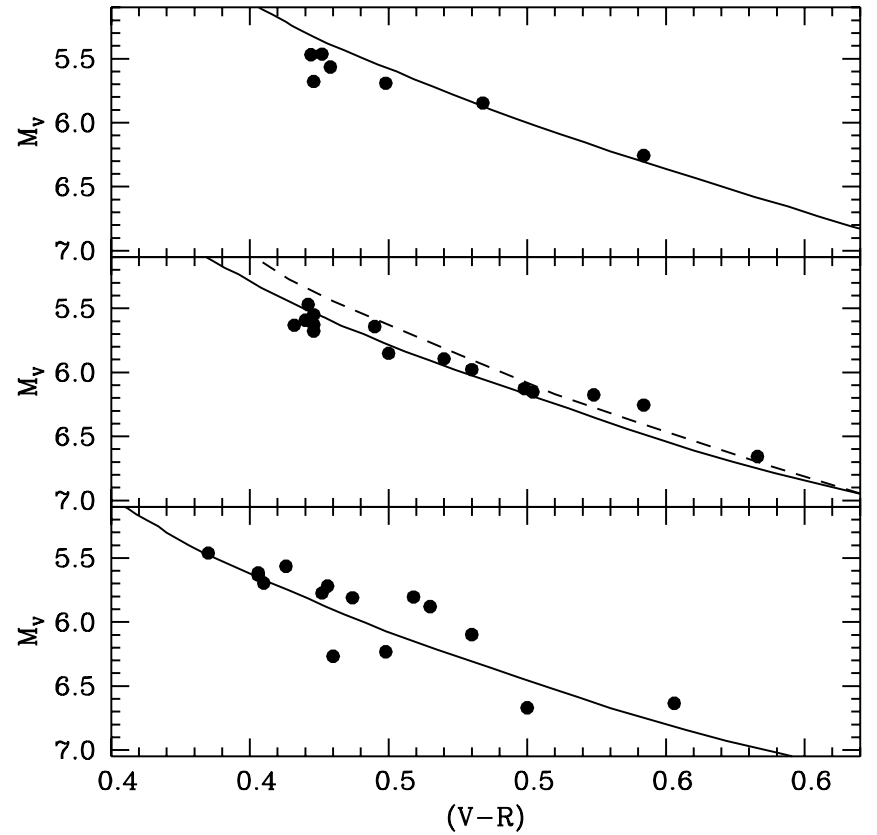

FIG. 16.-Same as Fig. 14, but for the $M_{V}-(V-R)$ CMD.

have been computed making use of the field dwarf sample by Percival et al. (2002), whose individual $[\mathrm{Fe} / \mathrm{H}]$ values are on the same scale of the open cluster metallicities by Gratton (2000). PS03 employed clusters' [Fe/H] estimates from Gratton (2000) and reddening from Twarog et al. (1997).

When we compare our models to the observed CMD of a cluster, we have chosen within our isochrone grid the one with metallicity closest to the cluster $[\mathrm{Fe} / \mathrm{H}]$. However, if no computed isochrone has a $[\mathrm{Fe} / \mathrm{H}]$ value within the quoted $1 \sigma$ error associated to the cluster one, we interpolate quadratically within our grid as previously discussed, in order to determine the isochrone with the precise cluster metallicity.

We consider a fit "successful" when the theoretical isochrones overlap the main cluster CMD branches for values of

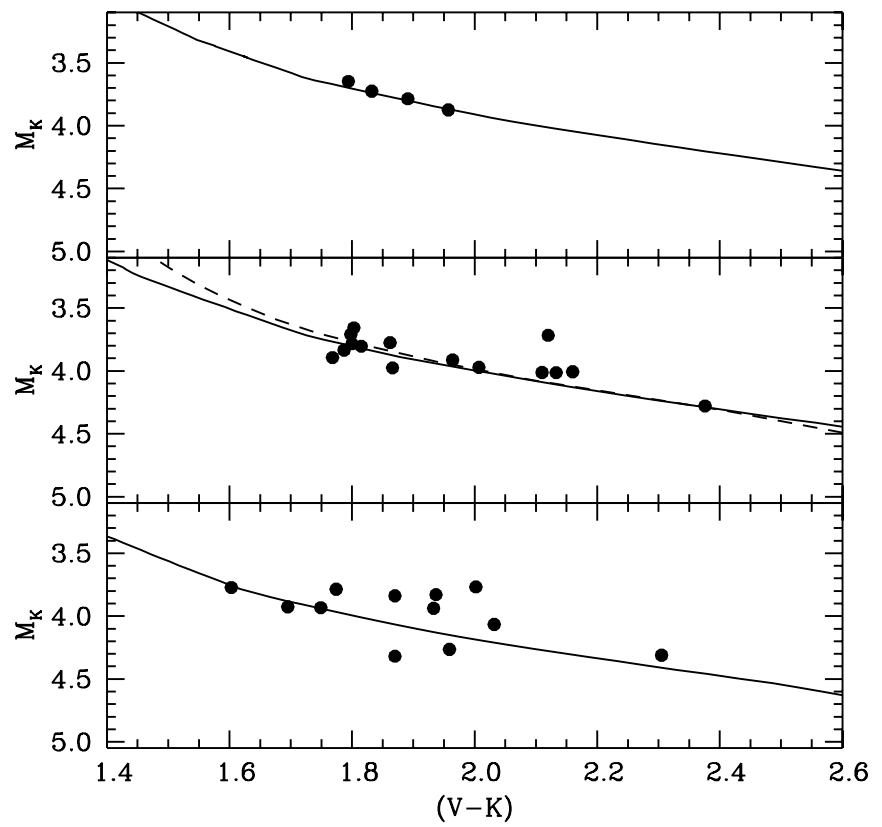

FIG. 17.-Same as Fig. 14, but for the $M_{K}-(V-K)$ CMD.

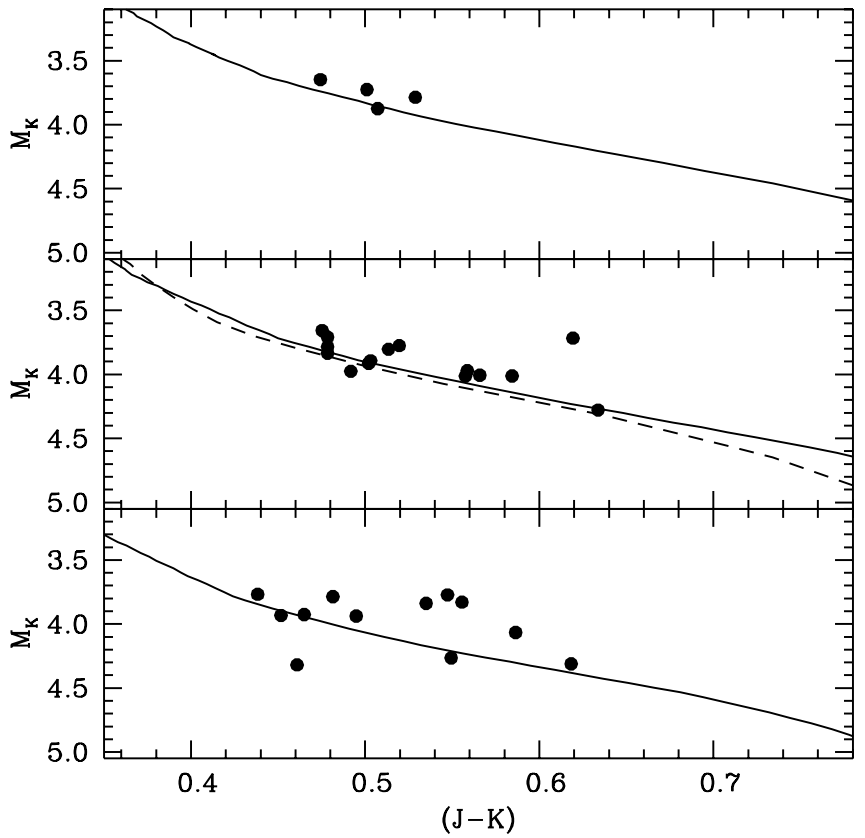

FIG. 18.-Same as Fig. 14, but for the $M_{K}-(J-K)$ CMD.

reddening, metallicity, and distance modulus in agreement with the empirical determinations.

Figures 19 and 20 display the comparison with the Hyades CMD corrected for the cluster distance; the cluster metallicity is $[\mathrm{Fe} / \mathrm{H}]=0.13$ according to Gratton (2000), and the reddening is zero. The observed CMD contains exactly the same stars in all three different photometric planes. The interpolated isochrone with $[\mathrm{Fe} / \mathrm{H}]=0.13$ that best matches the Hyades CMD has an age of $790 \mathrm{Myr}$ if overshooting is included, or 560 Myr for canonical models; note the match [that in $(B-V)$ and $(V-K)$ degrades only when $M_{V}>7.5$ ] of the unevolved
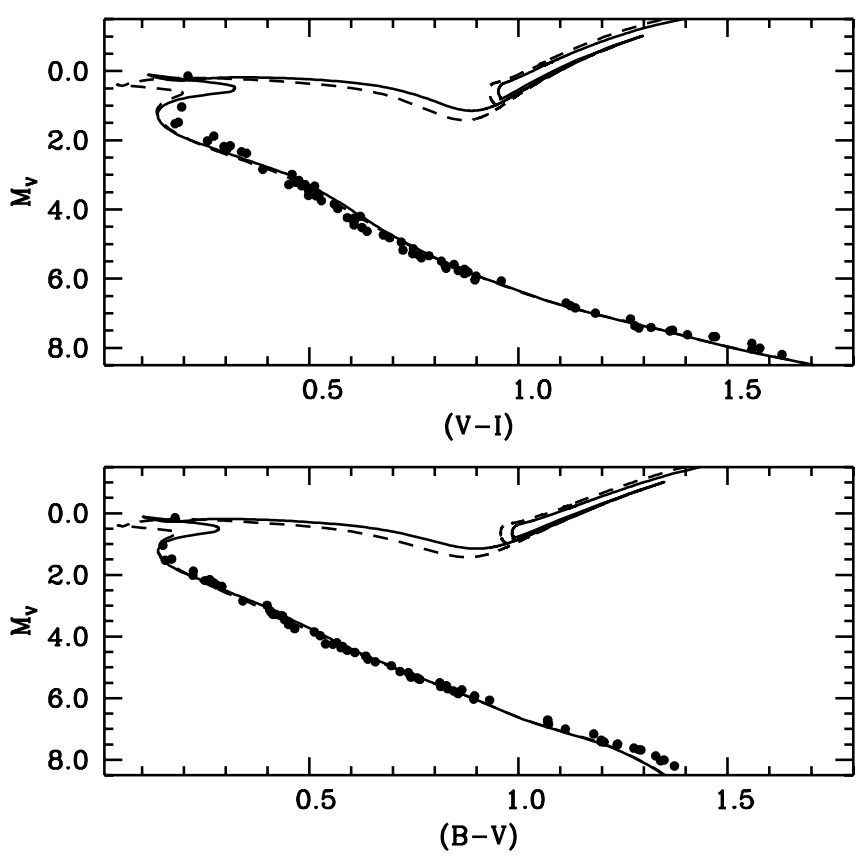

FIG. 19.-Comparison between our $[\mathrm{Fe} / \mathrm{H}]=0.13,790 \mathrm{Myr}$ overshooting (solid line) and $560 \mathrm{Myr}$ canonical (dashed line) isochrones and the Hyades $M_{V}-(B-V)$ and $M_{V}-(V-I) \mathrm{CMDs}$, corrected for the parallax distance of the individual stars. 


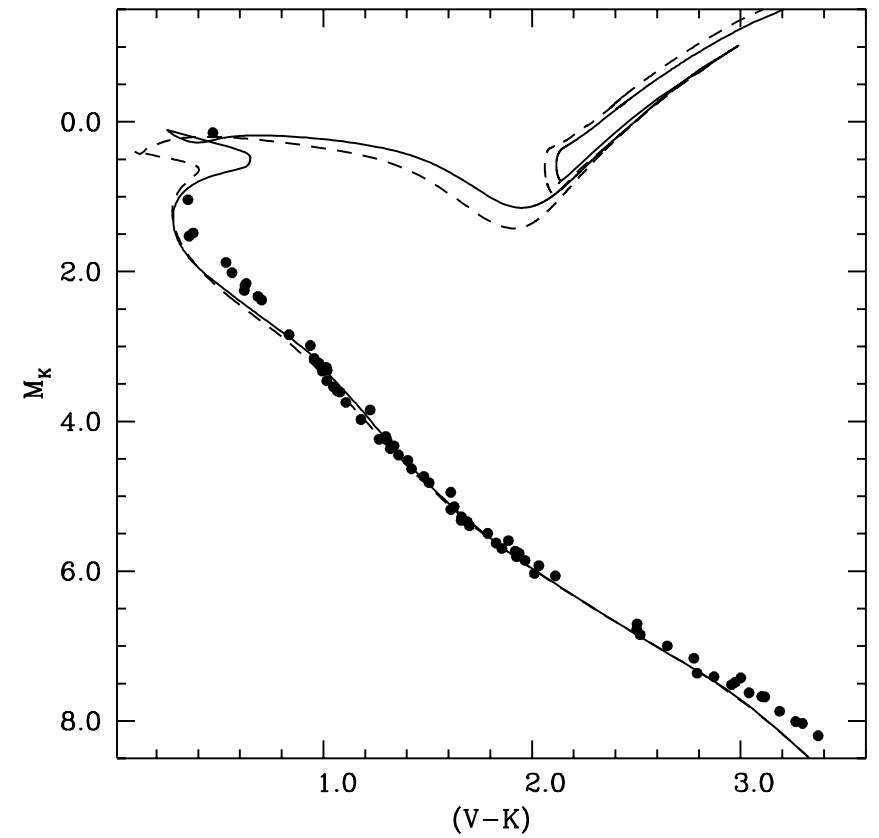

FIG. 20.- Same as Fig. 19, but for the $M_{K}-(V-K)$ CMD.

MS to the observed one, without applying any color shift. The TO region in $K-(V-K)$ is, however, less well reproduced with respect to the $B V I \mathrm{CMD}$, a fact that can be ascribed to either some inconsistencies in the color transformations, or photometric data, or a combination of both.

In case of NGC 2420, PS03 obtained $(m-M)_{0}=$ $11.94 \pm 0.07$, assuming $[\mathrm{Fe} / \mathrm{H}]=-0.44$ and $E(B-V)=$ $0.05 \pm 0.02$; our interpolated isochrones with $[\mathrm{Fe} / \mathrm{H}]=-0.44$ that best match the observed CMD provide a distance modulus $(m-M)_{0}=11.90$ and $E(B-V)=0.06$, in good agreement with the MS-fitting results (see Fig. 21). The age of the bestfitting isochron with overshooting is $3.2 \mathrm{Gyr}$, whereas the canonical one has an age of 2 Gyr. It is interesting to note that the isochrone with overshooting does provide a better match of the observed CMD TO region.

As for M67, PS03 have determined a distance modulus $(m-M)_{0}=9.60 \pm 0.09$, adopting $E(B-V)=0.04 \pm 0.02$ and $[\mathrm{Fe} / \mathrm{H}]=0.02 \pm 0.06$. Their MS-fitting determination was based on a different $B V I$ photometry (Montgomery et al. 1993); however, Sandquist (2004) has obtained exactly the same distance modulus with his photometry, employing the same method by PS03 and the same $[\mathrm{Fe} / \mathrm{H}]$ and $E(B-V)$. We fitted our $[\mathrm{Fe} / \mathrm{H}]=0.06$ isochrones to the $B V I$ cluster data and obtained a successful fit with $E(B-V)=0.02$ and $(m-M)_{0}=$ 9.66, in agreement, within the error bar, with the MS-fitting results. The result of the fit is shown in Figure 22. The MS and RGB of the cluster appear to be matched by both overshooting and canonical isochrones; also, the brightness of the He-burning stars is reproduced. It is also worth noting that Sandquist (2004, his Fig. 14) has shown how a number of isochrones in the literature are unable to fit the main location of the MS for $V>15.5-16$, when the empirical distance, reddening, and metallicity are considered; our isochrones, however, do provide a much better match to this MS region. The ages of the best-fitting isochrones are 4.8 and 3.8 Gyr for the overshooting and canonical models, respectively. As already noted by Sandquist (2004), who employed the Yi et al. (2001) and the Girardi et al. (2000) isochrones, the overshooting models do provide a worse fit to the TO region compared to the

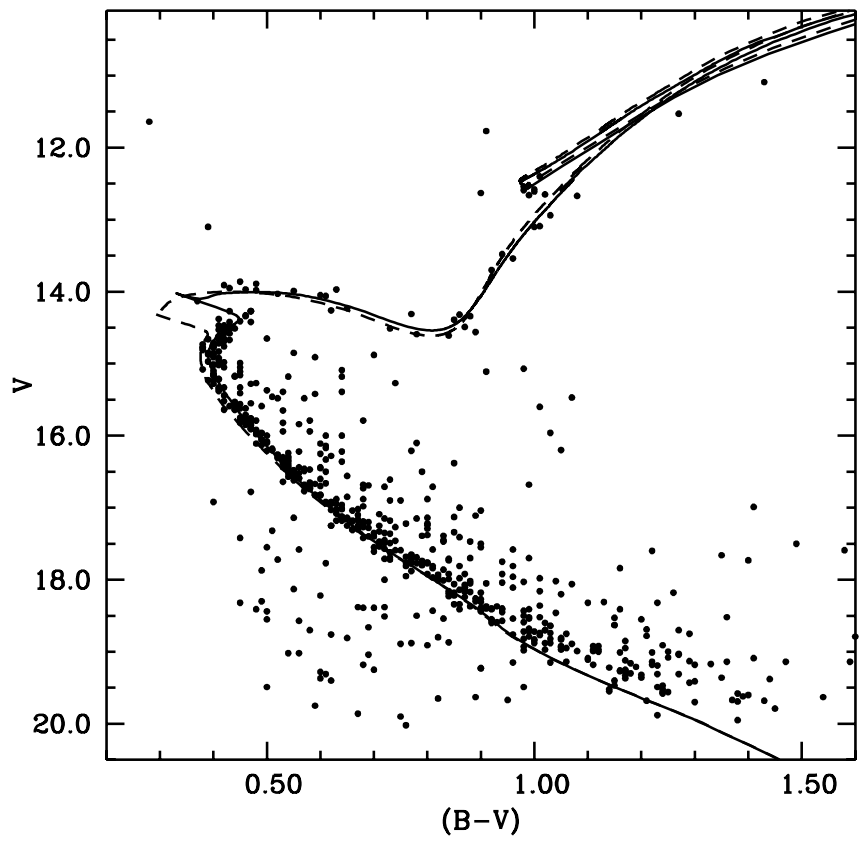

FIG. 21.-Comparison between our $[\mathrm{Fe} / \mathrm{H}]=-0.44,3.2$ Gyr overshooting (solid line) and 2 Gyr canonical (dashed line) isochrones, and NGC 2420 $V-(B-V) \mathrm{CMD}$. We have shifted the isochrones by $E(B-V)=0.06$ and $(m-M)_{0}=11.90$.

canonical ones. The mass evolving at the TO of the overshooting isochrone is equal to $\sim 1.2 M_{\odot}$; i.e., it is in the mass interval where the amount of overshooting is decreasing with mass. This means that M67 seem to indicate that the extension of the overshooting region has to be already almost down to zero for masses of $\sim 1.2 M_{\odot}$, at least for metallicities around solar. Figure 23 shows a comparison between the same isochrones displayed in Figure 22 and M67 CMDs in near-infrared
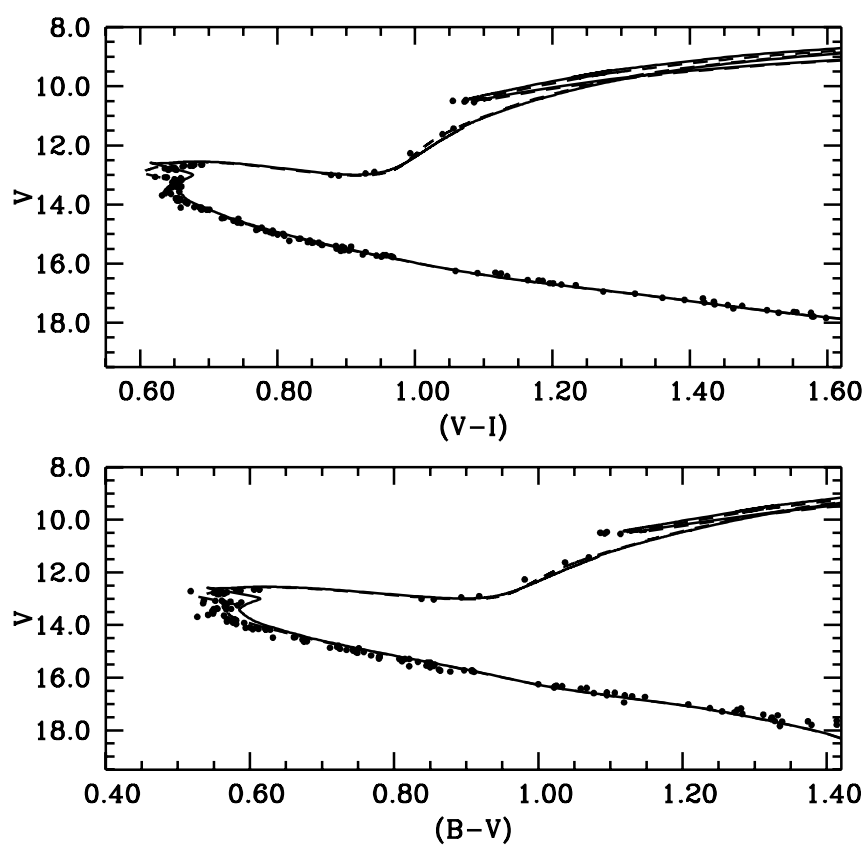

FIG. 22.-Comparison between our $[\mathrm{Fe} / \mathrm{H}]=0.06,4.8$ Gyr overshooting (solid line) and $3.8 \mathrm{Gyr}$ canonical (dashed line) isochrones, and M67 $V-(B-V)$ and $V-(V-I)$ CMDs. We have shifted the isochrones by $E(B-V)=0.02$ and $(m-M)_{0}=9.66$. 


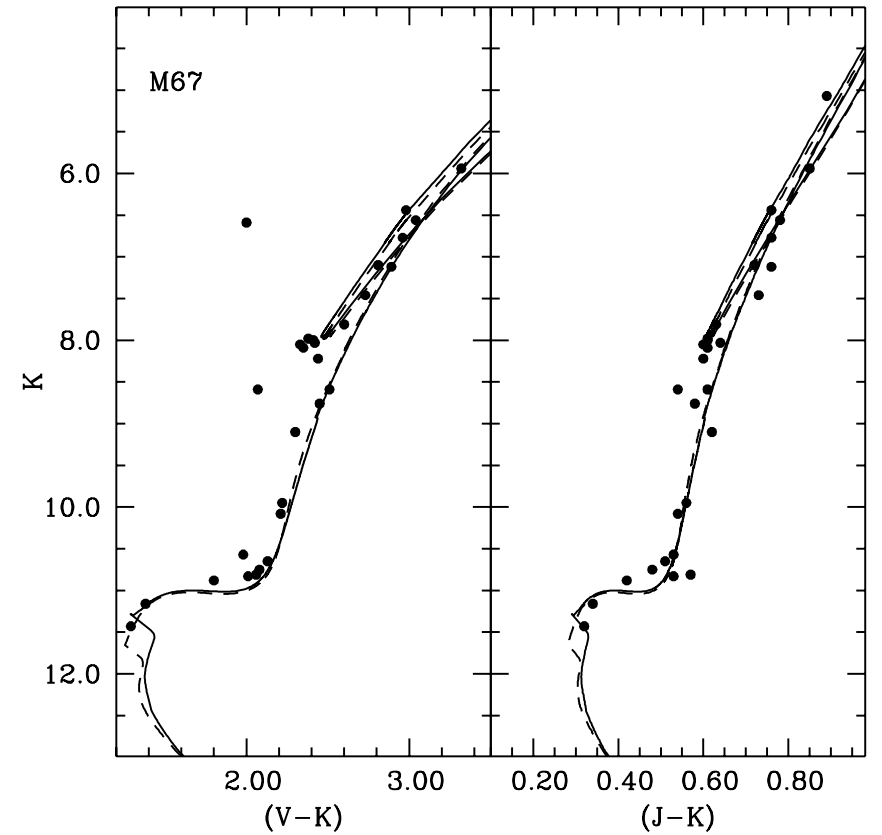

FIG. 23.- Same as Fig. 22, but for the $K-(V-K)$ and $K-(J-K)$ CMDs.

colors. Subgiants and RGB stars appear to be consistent with our isochrones for the same age, reddening, and distance modulus necessary to fit the $B V I$ data, with a small mismatch in the $K$-band absolute magnitude of He-burning stars.

PS03 have determined for NGC $188(m-M)_{0}=11.17 \pm$ 0.08 , by using $E(B-V)=0.09 \pm 0.02$ and $[\mathrm{Fe} / \mathrm{H}]=-0.03 \pm$ 0.06 . We fitted our interpolated $[\mathrm{Fe} / \mathrm{H}]=-0.03$ isochrones to the observed CMD, in both $M_{V}-(B-V)$ and $M_{V}-(V-I)$; the successful fit to the whole observed CMD is achieved for an age of $6.3 \mathrm{Gyr}$ (for both isochrones with and without overshooting), $E(B-V)=0.09$ and a distance modulus $(m-$ $M)_{0}=11.17$, coincident with the empirical MS-fitting result. Figure 24 shows the result of the fit with theoretical isochrones; the main location of the MS is reproduced, and one can also easily note that the isochrone with overshooting is only slightly different from the canonical one. This is due to the fact that the value of the mass evolving at the TO region is of about $1.16 M_{\odot}$, and for these masses the extension of the overshooting region is reducing to zero in our models. Thus, the comparison with NGC 188 does not contradict the possibility, raised by the comparison with M67, that the overshooting extension is already reduced to zero when the stellar mass is $\sim 1.2-1.3 M_{\odot}$ for metallicities around solar.

We have also compared our isochrones with the deep CMD by Kalirai et al. (2001) of NGC 6819, a cluster not employed by PS03 (see Fig. 25). We have adopted the recent $[\mathrm{Fe} / \mathrm{H}]=$ $0.09 \pm 0.03$ determination by Bragaglia et al. (2001) and found a a successful fit with our $[\mathrm{Fe} / \mathrm{H}]=0.06$ isochrones for $E(B-V)=0.14$ - in perfect agreement with the spectroscopic value $E(B-V)=0.14 \pm 0.04$ determined by Bragaglia et al. $(2001)$ - and a distance modulus $(m-M)_{0}=12.10$. The age of the cluster is 3 Gyr when overshooting is accounted for, or $1.8 \mathrm{Gyr}$ in case of canonical isochrones. The isochrones overlap the main locus of the cluster MS, and the brightness of the He-burning clump stars is also matched. In this case the isochrone with overshooting does provide a better match to the TO region.
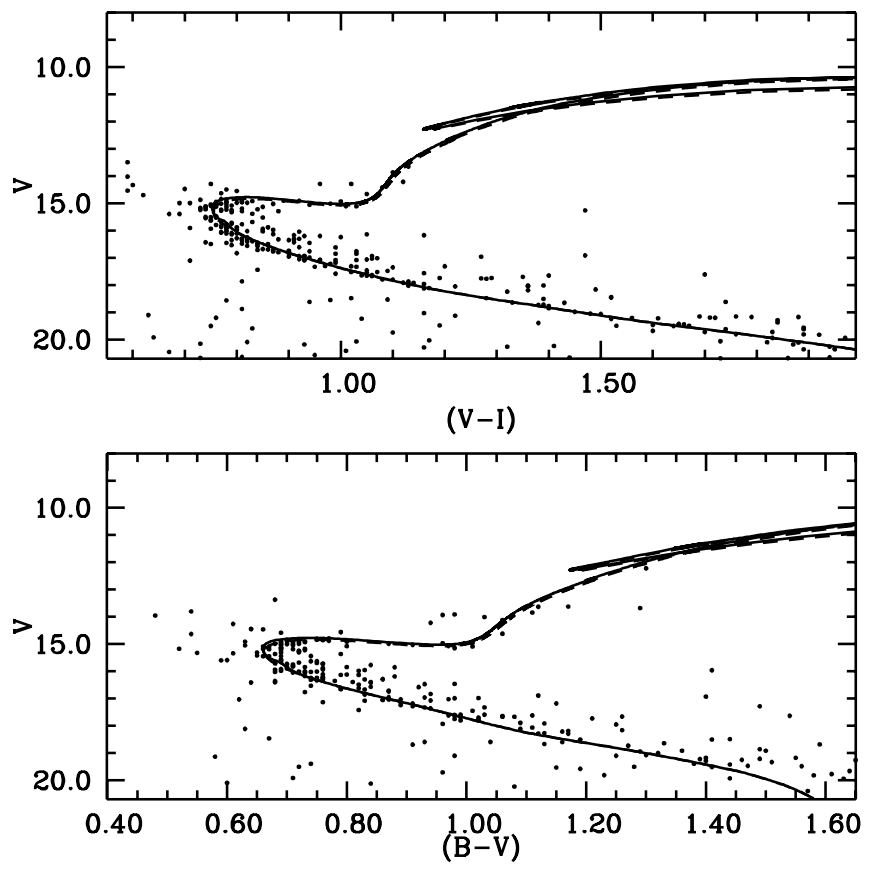

FIG. 24.-Comparison between our $[\mathrm{Fe} / \mathrm{H}]=-0.03,6.3$ Gyr overshooting (solid line) and canonical (dashed line) isochrones, and NGC $188 V-(B-V)$ and $V-(V-I)$ CMDs. We have shifted the isochrones by $E(B-V)=0.09$ and $(m-M)_{0}=11.17$.

\subsection{The Eclipsing Binary AI Phe}

The eclipsing binary star AI Phe is a particularly well-suited candidate to test the calibration of the mixing length adopted in our computations and the amount of overshooting from the convective core. The eclipsing binary analysis provides extremely precise values for the masses $(1.190 \pm 0.006$ and $\left.1.231 \pm 0.005 M_{\odot}\right)$ and radii $(1.762 \pm 0.007$ and $2.931 \pm$

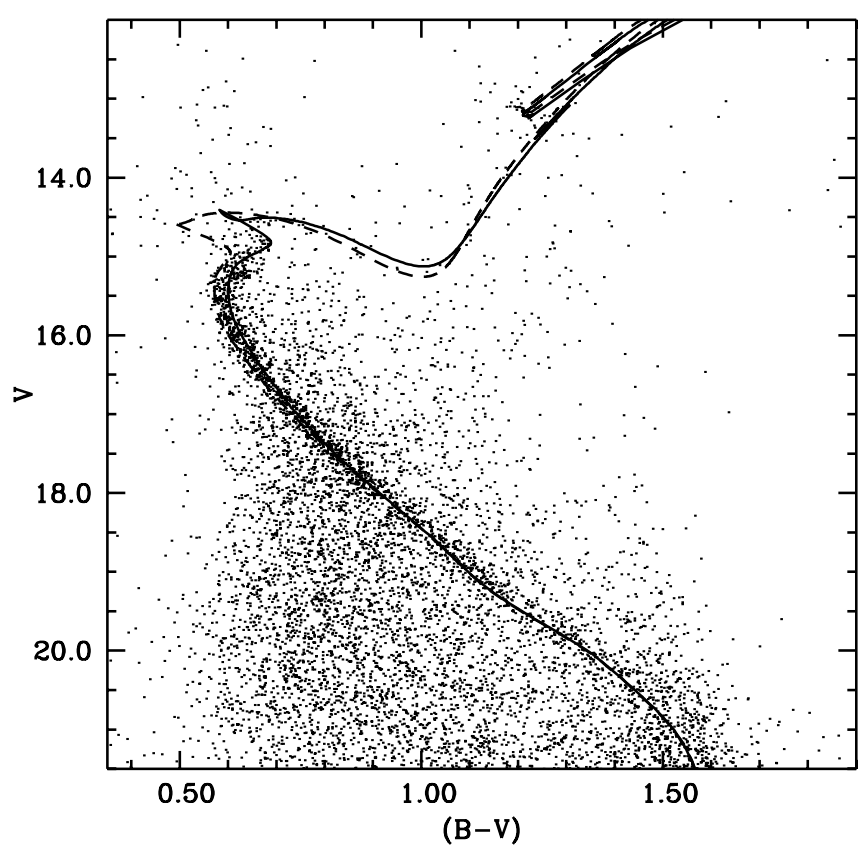

FIG. 25.-Comparison between our $[\mathrm{Fe} / \mathrm{H}]=0.06,3$ Gyr overshooting (solid line) and 1.8 Gyr canonical (dashed line) isochrones, and NGC 6819 $V-(B-V)$ CMD. We have shifted the isochrones by $E(B-V)=0.14$ and $(m-M)_{0}=12.10$. 


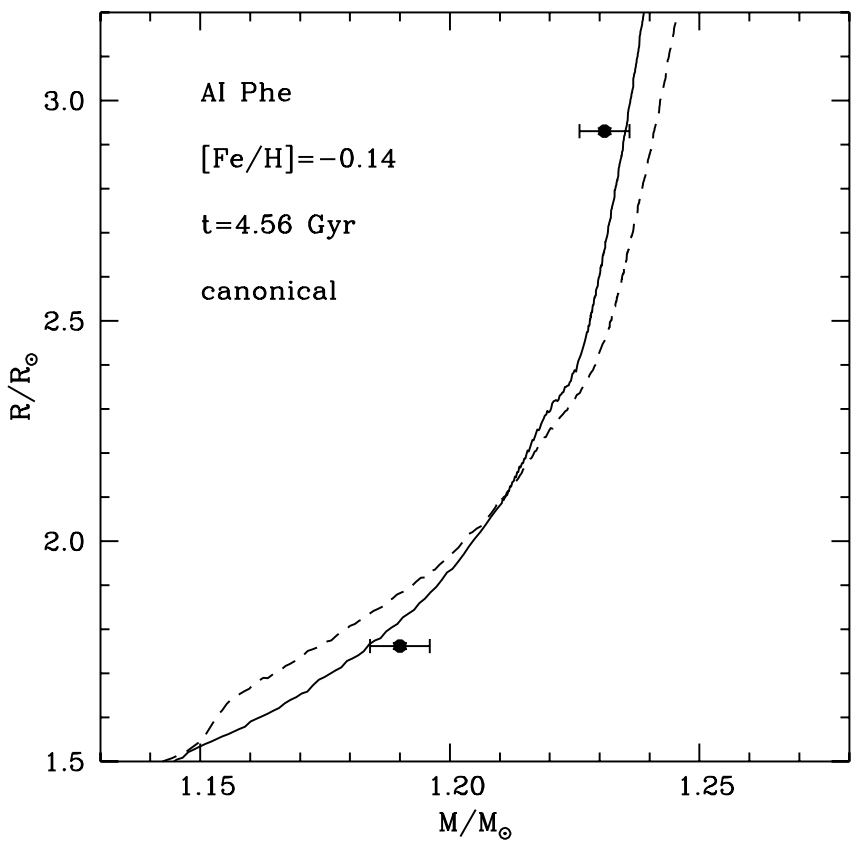

FIG. 26. - Comparison between the observed masses and radii of the components of the eclipsing binary AI Phe, and the predictions of a canonical isochrone (solid line) with the labeled metallicity and age. The dashed line represents an overshooting isochrone with the same metallicity and an age of 5.37 Gyr (see text for details).

$0.007 R_{\odot}$ ) of the two components (Milone et al. 1992); moreover, the metallicity of the system is estimated spectroscopically to be $[\mathrm{Fe} / \mathrm{H}]=-0.14 \pm 0.10$ by Andersen et al. (1988). The less massive component is located right after the TO region, at the beginning of the subgiant branch, whereas the more massive star is in a more advanced phase, close to the base of the RGB; this means that their radii are very sensitive to both the mixing-length calibration and overshooting treatment. In Figure 26 we display a comparison on the mass-radius plane between the observed values for the system, and the best-match theoretical isochron computed for $[\mathrm{Fe} / \mathrm{H}]=-0.14$ (quadratically interpolated among the available grid of metallicities). Both components can be fitted within the extremely small $1 \sigma$ error bars with the same age (approximately the solar age) and without including overshooting. Isochrones with overshooting cannot match simultaneously both components (an age of 5.37 Gyr provides the closest possible match to both components, which is, however, much worse than the case of canonical isochrones), even though, with our prescription, the overshooting region is about $0.08 H_{P}$ in this mass range. This result agrees with the case of M67, where we have shown (in agreement with the Sandquist 2004 results obtained by employing different isochrones) that masses of $\sim 1.2 M_{\odot}$ have an overshooting region already reduced to virtually zero for metallicities around solar.

\section{SUMMARY}

We have presented a homogeneous and updated database of stellar evolution models and isochrones for old, intermediate, and young stellar populations for a wide range of chemical compositions. Two large grids, with and without the inclusion of convective core overshooting during the H-burning phase, are provided. This set of theoretical models is suitable for population synthesis analysis as well as for investigating evolutionary properties of field and cluster stars in a wide range of masses and chemical compositions. The main points of this investigation can be summarized as follows:

1. Our models have been computed making use of many of the most recent updates of the stellar physics inputs. In particular, we employed new improved electron conduction opacities that cover the relevant physical conditions of electron degenerate cores along the RGB, new homogeneous reaction rates from the NACRE compilation, plus the latest determination for the important ${ }^{12} \mathrm{C}(\alpha, \gamma){ }^{16} \mathrm{O}$ reaction, new improved ATLAS9 color transformations and bolometric corrections, and a new EOS that covers homogeneously all the stellar structure along all the relevant evolutionary phases for the entire mass range covered by our computations. This EOS is in extremely good agreement with the OPAL EOS in the common domain of validity and eliminates the need to match the OPAL EOS to a different one when dealing with He burning and more advanced evolutionary stages.

2. The adopted initial He abundance for metal-poor models is in agreement with the most recent evaluations in this field obtained by CMB analysis; this value is also consistent with the estimate obtained from the $R$-parameter analysis on a large sample of Galactic globular clusters, performed with a theoretical calibration consistent with present models. This consistency is not achieved by the other available databases.

3 . The results of our calculations allow the computation of grids of isochrones covering a wide range of stellar ages, as well as the estimate of several astrophysical parameters such as the RGB transition mass, the He core mass at the He ignition, the amount of extra helium dredged up during RGB evolution, and the properties of models at the beginning of the thermal pulse phase on the AGB or at the carbon ignition.

4. We have also computed an additional large set of HB models - starting from a He core mass typical of ages around 13 Gyr-for all the metallicities available in our grid, which can be used to produce synthetic HB CMDs of various morphologies.

5. Stellar models and isochrones have been transferred to several observational CMDs by adopting updated bolometric corrections and color- $T_{\text {eff }}$ relations, computed specifically for this project, supplemented by empirical color transformation for the lower MS in selected color indices. The evolutionary tracks and isochrones are normalized in such a way that a simple interpolation on a point-by-point basis can be used in population synthesis codes to produce isochrones for metallicities intermediate among the values provided in the grid.

6. We have shown various comparisons with empirical data for unevolved field MS stars with available Hipparcos precise parallax measurements and metallicity estimates, and with data for open clusters with accurate photometry, metallicity estimates, and empirical MS-fitting distances. As a whole, one can note that a good agreement has been achieved between theory and observations.

7. We have carefully treated the way in which the core overshoot efficiency is decreased when approaching the radiative core regime in low-mass stars. Our analysis of the open cluster M67 and the eclipsing binary AI Phe shows that, at least around solar metallicities, core overshooting efficiency should be reduced to zero already for masses on the order of 1.2$1.3 M_{\odot}$. Further empirical tests should be performed in order to reach a definitive conclusion on this issue. We suggest always comparing results obtained with both canonical and overshooting models and isochrones for ages on the order of 4-5 Gyr. 
We have made a great effort in order to make available the whole theoretical framework to the scientific community in an easy and direct way through a dedicated Web site. We plan, in the near future, to prepare a Web interactive interface that will allow any potential user to compute isochrones, luminosity functions, synthetic CMDs, and integrated colors for any specified age and/or star formation history. The evolutionary database will soon be implemented with a self-consistent and extended set of theoretical models for an $\alpha$-enhanced heavyelement mixture, which will be presented in a forthcoming paper.

We warmly thank the editor, Brian Chaboyer, for his handling of the manuscript. We thank L. Girardi, S. Yi, and A. Potekhin for providing data in electronic form and for many insightful discussions, J. Kalirai for providing us with the photometry of NGC 6891, and S. Percival for making available to us the field star data in machine-readable form. We wish to warmly thank A. Irwin for all the help provided in using his EOS code, as well as for many interesting discussions. A special thanks to D. Vandenberg for sharing with us his evolutionary results in advance of publication. It is a pleasure to thank S. Degl'Innocenti for many useful discussions on helioseismology and standard solar models, and for providing the data for Figure 1. L. Piersanti is warmly acknowledged for the valuable suggestions provided during the updating the stellar evolution code. We are very grateful to M. Castellani for the relevant help provided when preparing the BASTI Web pages. S. C. is grateful for the hospitality at the Instituto de Astrofísica de Canarias in Tenerife. This research has made use of NASA's Astrophysics Data System Abstract Service and SIMBAD database operated at CDS, Strasbourg, France. This work was partially supported by MURST (PRIN2002, PRIN2003).
Alexander, D. R., \& Ferguson, J. W. 1994, ApJ, 437, 879

Allard, F., Hauschildt, P. H., Alexander, D. R., \& Starrfield, S. 1997, ARA\&A, 35,137

Alongi, M., Bertelli, G., Bressan, A., \& Chiosi, C. 1991, A\&A, 244, 95

Andersen, J., Clausen, J. V., Nordstrom, B., Gustafsson, B., \& Vandenberg, D. A. 1988, A\&A, 196, 128

Angulo, C., et al. 1999, Nucl. Phys. A, 656, 3

Anthony-Twarog, B. J., Twarog, B. A., Kaluzny, J., \& Shara, M. M. 1990, AJ, 99, 1504

Azusienis, A., \& Straižys, V. 1969, Soviet Astron., 13, 316

Bahcall, J. N., \& Pinsonneault, M. H. 1992, Rev. Mod. Phys., 64, 885 1995, Rev. Mod. Phys., 67, 781

Bahcall, J. N., Pinsonneault, M. H., \& Basu, S. 2001, ApJ, 555, 990

Barmina, R., Girardi, L., \& Chiosi, C. 2002, A\&A, 385, 847

Basu, S., Christensen-Dalsgaard, J., Perez Hernandez, F., \& Thompson, M. J. 1996, MNRAS, 280, 651

Bergbusch, P. A., \& Vandenberg, D. A. 1992, ApJS, 81, 163

2001, ApJ, 556, 322

Bessell, M. S. 1983, PASP, 95, 480 1990, PASP, 102, 1181

Bessell, M. S., \& Brett, J. M. 1988, PASP, 100, 1134

Bessell, M. S., Castelli, F., \& Plez, B. 1998, A\&A, 333, 231

Böhm-Vitense, E. 1958, Z. Astrophys., 46, 108

Bonifacio, P., et al. 2002, A\&A, 390, 91

Bono, G., Caputo, F., Cassisi, S., Castellani, V., \& Marconi, M. 1997, ApJ, 479, 279

Bono, G., Caputo, F., Cassisi, S., Marconi, M., Piersanti, L., \& Tornambé, A. 2000, ApJ, 543, 955

Bragaglia, A., Carretta, E., \& Gratton, R. G. 2001, AJ, 121, 327

Brocato, E., Cassisi, S., \& Castellani, V. 1998, MNRAS, 295, 711

Brocato, E., Castellani, V., Di Carlo, E., Raimondo, G., \& Walker, A. R. 2003, AJ, 125, 3111

Bruzual, G., \& Charlot, S. 2003, MNRAS, 344, 1000

Buser, R. 1978, A\&A, 62, 411

Caloi, V., D'Antona, F., \& Mazzitelli, I. 1997, A\&A, 320, 823

Caputo, F., Castellani, V., Chieffi, A., Pulone, L., \& Tornambé, A. 1989, ApJ, 340,241

Caputo, F., Chieffi, A., Castellani, V., Collados, M., Martínez-Roger, C., \&

Paez, E. 1990, AJ, 99, 261

Carpenter, J. M. 2001, AJ, 121, 2851

Cassisi, S. 2004, in ASP Conf. Ser. 310, Variable Stars in the Local Group, ed. D. W. Kurtz \& K. Pollard (IAU Colloq. 193; San Francisco: ASP), 489

Cassisi, S., Castellani, V., Degl'Innocenti, S., Salaris, M., \& Weiss, A. 1999, A\&AS, 134, 103

Cassisi, S., Castellani, V., Degl'Innocenti, S., \& Weiss, A. 1998, A\&AS, 129, 267

Cassisi, S., \& Salaris, M. 1997, MNRAS, 285, 593

Cassisi, S., Salaris, M., \& Irwin, A. W. 2003, ApJ, 588, 862

Castellani, V., Chieffi, A., Pulone, L., \& Tornambé, A. 1985, ApJ, 296, 204

Castellani, V., Degl'Innocenti, S., Fiorentini, G., Lissia, M., \& Ricci, B. 1997, Phys. Rep., 281, 309

Castellani, V., Degl'Innocenti, S., Marconi, M., Prada Moroni, P. G., \& Sestito, P. 2003, A\&A, 404, 645

Castelli, F. 1998, Mem. Soc. Astron. Italia, 69, 165

- 1999, A\&A, 346, 564

\section{EFERENCES}

Castelli, F., \& Cacciari, C. 2001, A\&A, 380, 630

Castelli, F., \& Kurucz, R. L. 1994, A\&A, 281, 817

2003, in Poster Papers, IAU Symp. 210, Modeling of Stellar Atmospheres, ed. N. E. Piskunov, W. W. Weiss, \& D. F. Gray (San Francisco: ASP)

Catelan, M., De Freitas Pacheco, J. A., \& Horvath, J. E. 1996, ApJ, 461, 231

Caughlan, G. R., \& Fowler, W. A. 1988, At. Data Nucl. Data Tables, 36, 411

Caughlan, G. R., Fowler, W. A., Harris, M. J., \& Zimmermann, B. A. 1985, At. Data Nucl. Data Tables, 32, 197

Chaboyer, B., \& Kim, Y.-C. 1995, ApJ, 454, 767

Cordier, D., Lebreton, Y., Goupil, M.-J., Lejeune, T., Beaulieu, J.-P., \& Arenou, F. 2002, A\&A, 392, 169

Cox, J. P., \& Giuli, R. T. 1968, Principle of Stellar Structure (London: Gordon $\&$ Breach)

Degl'Innocenti, S., Dziembowski, W. A., Fiorentini, G., \& Ricci, B. 1997, Astropart. Phys., 7, 77

De Santis, R., \& Cassisi, S. 1999, MNRAS, 308, 97

Dziembowski, W. A., Goode, Philip, R., Pamyatnykh, A. A., \& Sienkiewicz, R. 1995, ApJ, 445, 509

Dorman, B., \& Rood, R. T. 1993, ApJ, 409, 387

Freytag, B., \& Salaris, M. 1999, ApJ, 513, L49

Girardi, L., Bressan, A., Bertelli, G., \& Chiosi, C. 2000, A\&AS, 141, 371

Graboske, H. C., Dewitt, H. E., Grossman, A. S., \& Cooper, M. S. 1973, ApJ, 181,457

Gratton, R. G. 2000, in ASP Conf. Ser. 198, Euroconference on Stellar Clusters and Associations: Convection, Rotation, and Dynamos, ed. R. Pallavicini, G. Micela \& S. Sciortino (San Francisco: ASP), 225

Gratton, R. G., et al. 2001, A\&A, 369, 87

Green, E. M. 1988, in Calibration of Stellar Ages, ed. A.G. Davis Philip (Schenectady: Davis), 81

Grevesse, N., \& Noels, A. 1993, in Origin and Evolution of the Elements, ed. N. Prantzos, E. Vangioni-Flam, \& M. Cassé (Cambridge: Cambridge Univ. Press), 14

Grevesse, N., \& Sauval, A. J. 1998, Space Sci. Rev., 85, 161

Haft, M., Raffelt, G., \& Weiss, A. 1994, ApJ, 425, 222

Hayes, D. S. 1985, IAU Symp. 111, Calibration of Fundamental Quantities, ed. D. S. Hayes, L. E. Pasinetti, \& A. G. D. Philip (Dordrecht: Reidel), 225

Houdashelt, M. L., Bell, R. A., \& Sweigart, A. V. 2000, AJ, 119, 1448

Hubbard, W. B., \& Lampe, M. 1969, ApJS, 163, 297

Iglesias, C. A., \& Rogers, F. J. 1996, ApJ, 464, 943

Itoh, N., Hayashi, H., \& Kohyama, Y. 1993, ApJ, 418, 405 (I93)

Itoh, N., Mitake, S., Iyetomi, H., \& Ichimaru, S. 1983, ApJ, 273, 774

Johnson, H. L. 1965, ApJ, 141, 923

Johnson, H. L., Mitchell, R. I., Iriarte, B., \& Wisiniewski, W. Z. 1966, Commun. Lunar Panetary Lab., 4, 99

Kalirai, J. S., et al. 2001, AJ, 122, 266

Keller, S. C., \& Wood, P. R. 2002, ApJ, 578, 144

Krishna-Swamy, K. S. 1966, ApJ, 145, 174

Kunz, R., Fey, M., Jaeger, M., Mayer, A., Hammer, J. W., Staudt, G., Harissopulos, S., \& Paradellis, T. 2002, ApJ, 567, 643

Lamla, E. 1982, in Landolt-Börstein, Vol. VI/2b, ed. K. Schaifers \& H. H. Voigt (Berlin: Springer), 71

Lejeune, T., \& Schaerer, D. 2001, A\&A, 366, 538

Marigo, P., Bressan, A., \& Chiosi, C. 1996, A\&A, 313, 545 
Mazzitelli, I., D’Antona, F., \& Caloi, V. 1995, A\&A, 302, 382

Mermilliod, J.-C. 1992, Bull. Inf. Centre Donnees Stellaires, 40, 115

Meynet, G., \& Maeder, A. 2000, A\&A, 361, 101

Milone, E. F., Stagg, C. R., \& Kurucz, R. L. 1992, ApJS, 79, 123

Montgomery, K. A., Marschall, L. A., \& Janes, K. A. 1993, AJ, 106, 181

Munakata, H., Kohyama, Y., \& Itoh, N. 1985, ApJ, 296, 197

Percival, S. M., Salaris, M., \& Kilkenny, D. 2003, A\&A, 400, 541 (PS03)

Percival, S. M., Salaris, M., Van Wyk, F., \& Kilkenny, D. 2002, ApJ, 573, 174

Pinsonneault, M. H., Terndrup, D. M., Hanson, R. B., \& Stauffer, J. R. 2003, ApJ, 598, 588

Potekhin, A. Y. 1999, A\&A, 351, 787 (P99)

Potekhin, A. Y., Chabrier, G., \& Shibanov, Y. A. 1999, Phys. Rev. E, 60, 2193

Prather, M. J. 1976, Ph.D. thesis, Yale Univ.

Reimers, D. 1975, Mem. Soc. R. Sci. Liège, 8, 369

Renzini, A., Greggio, L., Ritossa, C., \& Ferrario, L. 1992, ApJ, 400, 280

Renzini, A., \& Ritossa, C. 1994, ApJ, 433, 293

Riello, M., Cassisi, S., Piotto, G., Recio-Blanco, A., De Angeli, F., Salaris, M., Pietrinferni, A., Bono, G., \& Zoccali, M. 2003, A\&A, 410, 553

Rocha-Pinto, H. J., Maciel, W. J., Scalo, J., \& Flynn, J. 2000a, A\&A, 358, 850

Rocha-Pinto, H. J., Scalo, J., Maciel, W. J., \& Flynn, J. 2000b, A\&A, 358, 869

Rogers, F. J., \& Nayfonov, A. 2002, ApJ, 576, 1064

Rogers, F. J., Swenson, F. J., \& Iglesias, C. A. 1996, ApJ, 456, 902

Roxburgh, I. W. 1992, A\&A, 266, 291

Salaris, M., \& Cassisi, S. 1996, A\&A, 305, 858 1998, MNRAS, 298, 166
Salaris, M., Cassisi, S., \& Weiss, A. 2002, PASP, 114, 375

Salaris, M., Degl'Innocenti, S., \& Weiss, A. 1997, ApJ, 484, 986

Salaris, M., Riello, M., Cassisi, S., \& Piotto, G. 2004, A\&A, 420, 911

Salaris, M., \& Weiss, A. 1998, A\&A, 335, 943 2002, A\&A, 388, 492

Sandquist, E. L. 2004, MNRAS, 347, 101

Schlattl, H., \& Salaris, M. 2003, A\&A, 402, 29

Schlegel, D. J., Finkbeiner, D. P., \& Davis, M. 1998, ApJ, 500, 525

Spergel, D. N., et al. 2003, ApJS, 148, 175

Spinrad, H., Dey, A., Stern, D., Dunlop, J., Peacock, J., Jimenez, R., \& Windhorst, R. 1997, ApJ, 484, 581

Sweigart, A. V., Greggio, L., \& Renzini, A. 1990, ApJ, 364, 527

Testa, V., Ferraro, F. R., Chieffi, A., Straniero, O., Limongi, M., \& Fusi Pecci, F. 1999, AJ, 118, 2839

Thoul, A. A., Bahcall, J. N., \& Loeb, A. 1994, ApJ, 421, 828

Twarog, B. A., Ashman, K. M., \& Anthony-Twarog, B. J. 1997, AJ, 114, 2556

VandenBerg, D. A., Swenson, F. J., Rogers, F. J., Iglesias, C. A., \& Alexander, D. R. 2000, ApJ, 532, 430

Von Hippel, T., \& Sarajedini, A. 1998, AJ, 116, 1789

Weiss, A., \& Salaris, M. 1999, A\&A, 346, 897

Woo, J.-H., \& Demarque, P. 2001, AJ, 122, 1602

Woo, J.-H., Gallart, C., Demarque, P., Yi, S., \& Zoccali, M. 2003, AJ, 125, 754

Yi, S., Demarque, P., Kim, Y.-C., Lee, Y.-W., Ree, C. H., Lejeune, T., \& Barnes, S. 2001, ApJS, 136, 417

Yi, S., et al. 2000, ApJ, 533, 670 